\title{
Rethinking dynamic capital structure models with roll-over debt*
}

\author{
Jean-Paul Décamps ${ }^{\dagger} \quad$ Stéphane Villeneuve $e^{\ddagger}$
}

This version: november 2011

\begin{abstract}
Dynamic capital structure models with roll-over debt rely on widely accepted arguments that have never been formalized. This paper clarifies the literature and provides a rigorous formulation of the equity holders' decision problem within a game theory framework. We spell out the linkage between default policies in a rational expectations equilibrium and optimal stopping theory. We prove that there exists a unique equilibrium in constant barrier strategies, which coincides with that derived in the literature. Furthermore, that equilibrium is the unique equilibrium when the firm loses all its value at default time. Whether the result holds when there is a recovery at default remains a conjecture.
\end{abstract}

Keywords strategic default. payoff dominant equilibrium. constrained optimal stopping time.

JEL subject classification: C61 . G33

*We thank Yann Braouezec, Darrell Duffie, Alexander Guembel, Monique Jeanblanc, Damien Lamberton, Thomas Mariotti, Sophie Moinas, Jean-Charles Rochet, Ekaterina Voltchkova and especially Hayne Leland and Chris Rogers for very helpful discussions, which led to this paper. We are also grateful to an anonymous associate editor and two referees whose insightful comments helped us to strongly improve the paper. We thank seminar participants at Stanford, Imperial College, Oxford and Bachelier, as well as at the Bachelier conference, London, July 2008; the international conference on price, liquidity and credit risks, Konstanz, November 2008; the symposium on optimal stopping with applications, Turku, June 2009. Financial support from the Agence Nationale de la Recherche (ANR-09-BLAN-0358-01) and the Chair: Marchés des risques et création de valeur, Fondation du Risque/SCOR is gratefully acknowledged. The paper was written when we were Academic Fellows of the Europlace Institute of Finance and we thank this institution for its hospitality. We remain solely responsible for the content of this paper.

†Toulouse School of Economics (CRM-IDEI), Manufacture des Tabacs, 21, Allée de Brienne, 31000 Toulouse, France. Email: decamps@cict.fr.

†Toulouse School of Economics (CRM-IDEI), Manufacture des Tabacs, 21, Allée de Brienne, 31000 Toulouse, France. Email: stephane.villeneuve@univ-tlse1.fr. 


\section{Introduction}

Structural models in corporate finance allow an integrated analysis of firm, debt and equity values, optimal capital structure and strategic default decisions. Particular attention has been devoted to the case where bankruptcy is endogenously triggered by limited liability equity holders who set the default time so as to maximize the value of their claim. The pioneering paper, Leland [18], solves the problem when the debt is modeled as a consol bond, meaning a commitment to pay coupons indefinitely at some constant coupon rate. In Leland [18], posing an infinite maturity for the debt guarantees a simple time homogenous setting in which the equity holders' problem takes the form of a standard perpetual American put option whose underlying asset is the value of the firm's asset, and the exercise price the present value of all coupon payments, net of tax shields. The optimal capital structure emerges from the tradeoff between bankruptcy costs and tax shields. Computations are explicit and the solution can be fully studied in an analytical setting. Structural models with infinite debt maturity have been extended in a number of contributions. Some important examples are Mella-Barral and Perraudin [24], Fan and Sundaresan [9], Goldstein, Ju and Leland [11], or Duffie and Lando [5] that extend Leland [18] in various thoughtful directions while maintaining the perpetual debt assumption.

To focus only on infinitely lived debt is clearly restrictive. Considering debt with finite maturity is, however, not a simple task as it breaks the stationary debt structure and precludes closed form solutions. In two influential papers, Leland [19] and Leland and Toft [22] circumvent this difficulty and propose a dynamic capital structure model with roll-over debt. The purpose is to study debt with arbitrary finite maturity and endogenous default in a time-homogenous environment that allows closed form solutions for the pricing of debt, firm and equity values. Specifically, in a capital structure model with roll-over debt, the firm's management pre-commits to retire debt of finite maturity continually and to replace it with a like amount of new debt in order to keep total coupon payments and principal constant at any instant of time. Because of this pre-commitment the literature agrees that firm, debt and equity cash flows must be time-independent. Then, resting on standard results on stopping theory within a time-homogenous setting, it is argued that a constant barrier strategy characterizes the optimal default policy. That is, default is triggered by the first time that the value of the firm's asset reaches a sufficiently low positive constant threshold. The optimal constant threshold is then derived by invoking the smooth pasting condition. This way of modeling the debt maturity has been widely used in the recent literature ${ }^{1}$ and is now presented in textbooks such as those of Lando [17] or Bielecki and Rutkowski [1]. However, and very surprisingly, the strategic default decision problem faced by equity holders in a capital structure model with roll-over debt has never been formulated properly and the

\footnotetext{
${ }^{1}$ Leland [20] extends Leland [18] and studies the role of debt maturity on the incentives of equity holders to increase the volatility of the firm's asset. Mauer and Ott [23] study in a setting à la Leland [18] the equity holders' decision to invest in a growth opportunity. Eom, Helwege and Huang [7] and Leland [21] examine yields spreads and default probabilities predictions across several structural models including Leland and Toft [22]. Hackbarth, Miao and Morellec [12] use Leland [18] to study the impact of macroeconomic conditions on credit risk and dynamic capital structure choice. Ericsson and Renault [8] rely on Leland and Toft [22] to develop a structural model with liquidity and credit risk. Hilberink and Rogers [14], Kyprianou and Surya [16], Chen and Kou [2] extend the Leland and Leland and Toft methodology to Lévy processes.
} 
proposed optimal default policy is always presented as 'natural' and 'clear' from previous results derived in the setting of time-homogenous models with infinite debt maturity. Given its large audience, we think it is important to correct some previous inaccuracies in this literature and therefore to allow academics to build on corporate models with roll-over debt with lucidity.

To clarify the issue, let us consider the seminal Leland and Toft [22] paper. In Leland and Toft [22], at any time the firm repays a nominal amount of $f d t$ and issues new debt with face value $f d t$, coupon $c d t$ and maturity $T$. At any instant of time the total amount of principal outstanding is therefore constant equal to $F \equiv f T$ and the total coupon to be paid continuously is $C \equiv c T$. The firm therefore maintains up to the default time a constant debt both in face value and coupon payments. The aggregate firm's debt structure is characterized by the triplet $(C, F, T)$. The total debt cash flow due to roll-over is $(C+f-d) d t$ where $d$ is the market price of the newly issued debt with face value $f d t$, coupon $c d t$ and maturity $T$. What can make the problem nonstationary? A first crucial observation is that each new debt is issued at market value and its price $d$ depends on the default policy. If the law of the default policy is time-dependent, then the market price of the newly issued debt will depend both on the current time and on the current value of the firm's asset and so will be the total cash raised through debt. It follows from this simple observation that the debt cash flow is not time-homogenous for any default policy as it is the case when the debt is a consol bond with cash flow simply equal to $C d t$. Keeping in mind this remark let us now turn to the equity holders' problem. Here is the second crucial observation. Equity holders, who have pre-committed to a debt structure characterized by the triplet $(C, F, T)$, choose a default policy and roll-over the debt until the default time. The actual equity cash flow is the sum of the payment flow generated from the firm's asset and the tax benefits minus the payment flow due to roll-over $(C+f-d) d t$. The key point is that, in this perfect information setting, by issuing debt in a current time interval $(0, d t)$ equity holders pin down the default time of the firm. Debt holders perfectly anticipate future equity holders' actions and will rationally refuse to buy the newly issued debt in time interval $(0, d t)$ if they recognize that equity holders have incentives to deviate at future dates from the default policy chosen at date 0 . The resulting rational expectations equilibrium problem requires a definition and a characterization of equilibrium default policies. Then the equity holders' decision problem is to select an equilibrium default policy that maximizes at date 0 the sum of the expected discounted equity cash flow. The main source of confusion in the literature is that this equilibrium problem, although clearly suggested by Leland and Toft $[22]^{2}$, has surprisingly never been written and studied. On the contrary, when focusing on the equity value, the literature does not consider the actual equity cash flow but writes the equity value as the difference between the firm value and the currently outstanding debt. The value of the currently outstanding debt, by definition, takes into account all the cash flow due to roll-over up to the current date but does not take into account that new debt will be issued and withdrawn beyond that current date. It is therefore not clear whether or not the expression for the equity value used in the literature accounts for all the cash flows due to roll-over up to the default. In addition,

\footnotetext{
${ }^{2}$ Leland and Toft [22] write page 989 “ (the default triggering) $V_{B}$ will be determined endogenously and shown to be constant in a rational expectations equilibrium." This important point is not explained and the equilibrium problem is not written.
} 
as already said, the previous studies restrict the set of default policies to constant barrier strategies. Thus, the following questions drive our analysis: What is the precise formulation of the equity holders' problem in a dynamic capital structure model with roll-over debt? What does a full characterization of equilibria look like? Is a dominant equilibrium existing?

Addressing these issues allow us to provide a general and rigorous presentation of capital structure models with roll-over debt. Our main results are as follows. We justify the particular representation of the equity value used in the previous studies. Our results hold for any capital structure model with roll-over debt and do not involve any ad hoc restrictions on the default policies. We formulate for the first time the equity holders' problem. It aims at selecting a payoff dominant equilibrium in the sense of Harsanyi and Selten [13]. We spell out the linkage between constant barrier policies in a rational expectations equilibrium and optimal stopping theory. We prove that there exists a unique equilibrium in constant barrier strategies, which coincides with that derived in the literature. Furthermore, that equilibrium is the unique equilibrium when the firm loses all its value at default time. Whether the result holds when there is a recovery at default remains a conjecture.

The outline of the paper is as follows. Section 2 reviews the literature on debt structure and optimal default and poses the questions raised by the treatment of these models in the existing studies. Section 3 formulates the equity holders' problem. Section 4 explains the results posed in the literature and establish the existence and uniqueness of equilibrium in barrier strategies. Section 5 discusses our results and investigates extensions of our study. Section 6 concludes.

\section{Debt structure and optimal default}

Following standard exposition of structural models, we consider that equity and debt can be viewed as contingent claims on the asset of the firm. The price dynamics under the risk neutral probability measure $\mathbb{Q}$ evolves as

$$
d V_{s}=(r-\delta) V_{s} d s+\sigma V_{s} d B_{s} .
$$

The process $\left(B_{s}\right)_{s \geq 0}$ is a standard Brownian motion with respect to its own filtration $\left(\mathcal{F}_{s}\right)_{s \geq 0}$ that models the flow of information. The firm generates cash flow at the rate $\delta V_{s}$ at time $s$ for some constant $\delta \in(0, \infty)$. The constant $r$ denotes the riskless interest rate and the discounted value $e^{-(r-\delta) s} V_{s}$ of the firm's asset process is a martingale under $\mathbb{Q}$, that is

$$
\mathbb{E}\left[e^{-(r-\delta)(u-s)} V_{u} \mid \mathcal{F}_{s}\right]=V_{s},
$$

for all $0 \leq s \leq u$. We use throughout the paper the following remarks and notations. The strong solution of (1) with $V_{t}=x$ is

$$
V_{t+s}=x \exp \left[\left(r-\delta-\frac{\sigma^{2}}{2}\right) s+\sigma W_{s}\right]
$$

where $\left(W_{s} \stackrel{\text { def }}{=} B_{t+s}-B_{t}\right)_{s \geq 0}$ is a standard Brownian motion $\left(\mathcal{F}_{t+s}\right)_{s \geq 0}$ adapted. We denote by $\mathcal{T}_{t, \infty}$ the set of $\left(\mathcal{F}_{t+s}\right)$-stopping times with values in $[t, \infty]$. Using Equation $(2)$, we note 
that every $\tau_{t} \in \mathcal{T}_{t, \infty}$ can be expressed as $\tau_{t}=t+\tau \circ \Theta_{t}$ where $\tau \in \mathcal{T}_{0, \infty}$ and $\Theta_{t}$ is the shift operator $^{3}$. Moreover, for all $t \geq 0$, we note $\mathbb{Q}_{t, x}$ the probability measure on $\left(\Omega,\left(\mathcal{F}_{t+s}\right)_{s \geq 0}\right)$ under which $V_{t}=x$. We have for any $\phi$ bounded measurable function

$$
\mathbb{E}\left(\phi\left(V_{\tau_{t}}\right) \mid \mathcal{F}_{t}\right)=\mathbb{E}_{t, x}\left(\phi\left(V_{\tau}\right)\right)
$$

Structural models with endogenous default are discussed in many textbooks. We refer to Lando [17] page 60 and also to Section 2.1 of Duffie and Lando [5] for a review of this approach initiated by Leland [18]. In these models, the firm, say the owner of the firm's asset at date 0 , issues debt so as to take advantage of tax shields offered for interest expense. The debt is sold and the proceeds from the sale are paid at date 0 as a cash distribution to initial equity holders. Equity holders select strategically the default policy at date 0 after debt being in place in order to maximize the value of their claim. The primary concern of this paper is the determination of the strategic default when the firm pre-commits to roll-over its debt as in the seminal paper of Leland and Toft [22].

\subsection{Debt with infinite maturity}

We start with the strategic default policy when the firm is partly financed by debt with infinite maturity. We refer to Duffie [4] page 264 or again to section 2.1 of Duffie and Lando [5] for a detailed presentation. In this standard setting, debt with infinite maturity pays continuously a tax deductible coupon $C$. The firm generates an income stream of $\left(\delta V_{s}+\theta C\right) d s$ until default where $\theta$ denotes the corporate tax. At default time $\tau$, a fraction $\alpha \in[0,1]$ of the assets are lost as a frictional cost and debt holders get the rest of the value $(1-\alpha) V_{\tau}$. Therefore, at time 0 , for a given default policy $\tau$, the firm and debt values are respectively

$$
v(0 ; \tau)=\mathbb{E}\left[\int_{0}^{\tau} e^{-r s}\left(\delta V_{s}+\theta C\right) d s+(1-\alpha) e^{-r \tau} V_{\tau}\right]
$$

and

$$
D(0 ; \tau)=\mathbb{E}\left[\int_{0}^{\tau} e^{-r s} C d s+(1-\alpha) e^{-r \tau} V_{\tau}\right]
$$

Note that the difference of values between the firm and the firm's asset is only due to taxation and losses at default. The equity cash flow is time-independent and formed by the difference between the firm cash flow and the debt cash flow up to default time. The value of equity at time 0 is

$$
E(0 ; \tau)=\mathbb{E}\left[\int_{0}^{\tau} e^{-r s}\left(\delta V_{s}-(1-\theta) C\right) d s\right] .
$$

It is important to stress that no assumptions are made on stopping time $\tau$. In particular it is not assumed that the equity value is a simple function of the current value of the firm's asset $V_{0}$. This property however holds and is part of the solution to the equity holders' problem that we now describe.

\footnotetext{
${ }^{3}$ See for instance Revuz and Yor [26] page 36 for the definition of the shift operator.
} 
The equity holders' only decision is to select the default policy that maximizes the value of their claim. Equity holders take this decision strategically after debt being in place and therefore chose the default time $\tau$ that solves the stopping problem

$$
\sup _{\tau \in \mathcal{T}_{0, \infty}}(v(0 ; \tau)-D(0 ; \tau))
$$

or equivalently

$$
\sup _{\tau \in \mathcal{T}_{0, \infty}} \mathbb{E}\left[\int_{0}^{\tau} e^{-r s}\left(\delta V_{s}-(1-\theta) C\right) d s\right] .
$$

Note that, for any default policy $\tau \in \mathcal{T}_{0, \infty}$, the equity cash flow $\left(\delta V_{s}-(1-\theta) C\right) d s$ does not change as a function of $s$ but simply varies with the current value of the firm's asset $V_{s}$. It can be shown from this time-homogenous property that the optimal stopping time solution to (6) is unsurprisingly a barrier strategy $\tau_{B}=\inf \left\{s: V_{s} \leq B\right\}$ where $B$ is a positive constant. It then follows that the equity value solution to (6) is indeed a function of the current value of the firm's asset $V_{0}$. Furthermore, the optimal barrier $B_{\infty}$ is determined by the classical smooth-pasting condition

$$
\frac{\partial}{\partial V}\left(v\left(V, \tau_{B}\right)-D\left(V, \tau_{B}\right)\right)_{\mid V=B}=0
$$

that says that the value function associated with stopping problem (6) is of class $C^{1}$ across the optimal boundary. ${ }^{4}$ We show thereafter that the problem of the endogenous default timing is very different in a model with roll-over debt and that the above reasoning cannot be applied. A crucial reason is that, in a dynamic capital structure model with roll-over debt, the equity cash flow is not time-homogenous for any default policy $\tau \in \mathcal{T}_{0, \infty}$.

\subsection{Capital structure models with roll-over debt: summary and questions}

We now present capital structure models with roll-over debt. The seminal papers are those of Leland [19] and Leland and Toft [22]. We draw on Hilberink and Rogers [14] who propose a very clear and unified setting for these models.

In corporate models with roll-over debt, the firm pre-commits to retire and issue debt at any time according to a so called maturity profile defined by a probability measure $\mu$ whose tail function will be denoted by

$$
\Phi(x)=\int_{x}^{+\infty} \mu(d y)
$$

The rule with which the firm repays and renews debt is as follows. In any time interval $(t, t+d t)$, the firm issues new debt with face value $f d t$. In any time interval $(t+s, t+s+d s)$

\footnotetext{
${ }^{4}$ Remark that in Equation (7), the firm and debt values (3) and (4) are written as functions of the current value of the firm's asset $V$. This holds because the stopping time $\tau_{B}$ considered in $(7)$ is a constant barrier strategy.
} 
the firm pays back $f d t(\Phi(s)-\Phi(s+d s)) \equiv-f d t d \Phi(s)$. This implies that the amount of money the firm has to pay back in $(t, t+d t)$ due to all debt issued up to current time 0 is

$$
\begin{aligned}
\left(\int_{-\infty}^{0}-f d v d \Phi(t-v)\right) & =-d\left(\int_{-\infty}^{0} f d v \Phi(t-v)\right) \\
& =-d\left(\int_{t}^{\infty} f \Phi(u) d u\right) \\
& =f \Phi(t) d t
\end{aligned}
$$

Taking $t=0$ in (8), we see that the amount to be paid back in time interval $(0, d t)$ due to all pending debt at time $t=0$ is $f d t$, the same as the face value of the newly-issued debt in $(0, d t)$. Thus, at each instant of time, the face value of all pending debt is constant, equal to

$$
F=f \int_{0}^{\infty} \Phi(t) d t
$$

Now, each newly issued debt (with principal $f d t$ ) pays coupons at rate $c$ until default. In any time interval $(t, t+d t)$ the market value of newly issued debt is $d(t ; \tau) d t$ with

$$
d(t ; \tau)=f \int_{0}^{\infty} b(t, t+u ; \tau)[-d \Phi(u)]
$$

where $b(t, t+u ; \tau)$ is the value of a bond issued at time $t$ with coupon rate $c$, face value 1 , maturity $t+u$ and default time $\tau$. That is we have

$$
\begin{aligned}
b(t, t+u ; \tau)= & \mathbb{E}\left[\int_{t}^{(t+u) \wedge \tau} c e^{-r(s-t)} d s \mid \mathcal{F}_{t}\right]+\mathbb{E}\left[e^{-r u} \mathbb{1}_{\tau \geq t+u} \mid \mathcal{F}_{t}\right] \\
& +\frac{1}{F}(1-\alpha) \mathbb{E}\left[V_{\tau} e^{-r(\tau-t)} \mathbb{1}_{\tau<t+u} \mid \mathcal{F}_{t}\right]
\end{aligned}
$$

The first term on the right of (10) is interpreted as the net present value of all coupons paid up to date $t+u$ or default time $\tau$, whichever is sooner. The second term is the net present value of the principal repayment, if it occurs before bankruptcy. The final term is the net present value of what is recovered upon default, if this happens before maturity. In line with the literature, the fraction of firm asset value lost in bankruptcy is $\alpha$ and the remaining value $(1-\alpha) V_{\tau}$ is distributed to debt holders. Of this, the debt holder with face value 1 gets the fraction $\frac{1}{F}$, since his debt represents this fraction of the total debt outstanding.

Note that, taking into account all previously issued debt, the aggregate coupon paid in $(t, t+d t)$ is

$$
\int_{-\infty}^{0}-f c d v d \Phi(t-v)=f c \Phi(t) d t
$$

It follows that the total coupon $C$ paid at any time is

$$
C=\int_{0}^{\infty} f c \Phi(t) d t=c F
$$


Finally, remark that the expected maturity of each newly issued debt satisfies the relation

$$
\int_{0}^{\infty} t \mu(d t)=\int_{0}^{\infty} \Phi(u) d u
$$

We shall assume thereafter that the maturity profile $\mu$ is such that $\bar{\Phi}(0) \equiv \int_{0}^{\infty} \Phi(u) d u<\infty$. In words, the debt profile has a finite expected maturity.

To summarize, the aggregate debt structure on which the firm pre-commits is fully characterized by the triplet $(C, F, \mu)$. By rolling-over the debt according to the maturity profile $\mu$, the firm maintains at any time before default a constant debt level both in face value and coupon payments. The concept of maturity profile has been introduced by Hilberink and Rogers [14] and allows a general presentation of models with roll-over debt. Specifically, the case $\mu(d s)=\delta_{T}(d s)$ where $\delta_{T}$ is the Dirac measure at $T$ corresponds to the Leland and Toft [22] model where each new debt is issued with maturity $\bar{\Phi}(0)=\int_{0}^{\infty} s \mu(d s)=\int_{0}^{\infty} s \delta_{T}(d s)=T$. The case $\mu(d s)=m e^{-m s} d s$ with $m>0$ corresponds to the exponential model of Leland [18] where the maturity of each new debt is chosen randomly according to an exponentially distributed random variable with mean $\bar{\Phi}(0)=\frac{1}{m}$.

Always following this well established literature, we now write $D(0 ; \tau)$, the value of all outstanding debt at time 0 :

$$
\begin{aligned}
D(0 ; \tau)= & \int_{0}^{\infty} f b(0, t ; \tau) \Phi(t) d t \\
= & f c \mathbb{E}\left[\int_{0}^{\tau} e^{-r s} \int_{s}^{\infty} \Phi(u) d u d s\right]+f \mathbb{E}\left[\int_{0}^{\tau} e^{-r s} \Phi(s) d s\right] \\
& +\frac{(1-\alpha) f}{F} \mathbb{E}\left[V_{\tau} e^{-r \tau} \int_{\tau}^{\infty} \Phi(u) d u\right]
\end{aligned}
$$

which simplifies to

$$
\begin{aligned}
D(0 ; \tau) & =\mathbb{E}\left[\int_{0}^{\tau} e^{-r s} f(c \bar{\Phi}(s)+\Phi(s)) d s+\frac{(1-\alpha) f}{F} V_{\tau} e^{-r \tau} \bar{\Phi}(\tau)\right] \\
& =\mathbb{E}\left[\int_{0}^{\tau} e^{-r s} \frac{1}{\bar{\Phi}(0)}(C \bar{\Phi}(s)+F \Phi(s)) d s+(1-\alpha) V_{\tau} e^{-r \tau} \frac{\bar{\Phi}(\tau)}{\bar{\Phi}(0)}\right]
\end{aligned}
$$

where $\bar{\Phi}(s) \equiv \int_{s}^{\infty} \Phi(u) d u$.

Equation (12) accounts for the future debt cash flow that is extant at time 0. Accordingly, in Equation (12), the coupon and the remaining principal of currently-extant debt decline with time. The same remark applies to the total payment at bankruptcy time $\tau$. Formally, the maturity profile of the existing debt at any moment is described by $\frac{\Phi(t)}{\bar{\Phi}(0)} d t$.

There is a corporate tax rate $\theta$, and the total coupon $C$ paid at any time can be offset against tax. The corporate $\operatorname{tax} \theta$ does not lead to a riskless debt and we consider thereafter that

$$
\int_{0}^{\infty} e^{-r s} \frac{1}{\bar{\Phi}(0)}(C \bar{\Phi}(s)+F \Phi(s)) d s>\int_{0}^{\infty} e^{-r s} \theta C d s=\frac{\theta C}{r}
$$


where the left hand side of Equation (13) is deduced from Equation (12) and represents the value of a riskless roll-over debt. ${ }^{5}$ Therefore, for any default policy $\tau$, the firm cash flow is time-homogenous and is equal to $\left(\delta V_{s}+\theta C\right) d s$. The firm value has thus a similar representation to the one derived when the debt has an infinite maturity, that is

$$
v(0 ; \tau)=\mathbb{E}\left[\int_{0}^{\tau} e^{-r s}\left(\delta V_{s}+\theta C\right) d s+(1-\alpha) e^{-r \tau} V_{\tau}\right] .
$$

Based on the results obtained when the debt maturity is infinite, the literature agrees ${ }^{6}$ to restrict the set of default policies to constant barrier strategies $\tau_{B}=\inf \left\{s: V_{s} \leq B\right\}$; to define the value at time 0 of the firm's equity as

$$
E\left(0 ; \tau_{B}\right)=v\left(0 ; \tau_{B}\right)-D\left(0 ; \tau_{B}\right)
$$

and to invoke the smooth-pasting condition (7) to determine the optimal bankruptcy threshold $B^{*}$. This treatment of models with roll-over debt raises several questions. Let us closely examine the above statements that are taken for granted by the literature. Three interrelated remarks drive our analysis.

1. Does Equation (15) account for all the cash flow due to roll-over? The firm value (14) clearly accounts for all the firm's cash flow up to the default time $\tau$. This is however not the case for the debt value (12) that only accounts for the future debt cash flow that is extant at time 0 . Precisely, $D(0 ; \tau)$ is the debt value at current time 0 and does not take into account that new debt will be issued and withdrawn beyond that date. In a model with roll-over debt, the net cash flow rate to debt at any future time $s$ is on the set $\{\tau>s\}$ the sum of the total coupon $C$, plus the retirement of principal $f$, and less $d(s ; \tau)$, the instantaneous market value of the newly issued bond. Therefore, taking into account all cash inflow and cash outflow due to roll-over, the value at date 0 of the total debt for a given default policy $\tau$ is

$$
D_{t o t}(0 ; \tau)=\mathbb{E}\left[\int_{0}^{\tau} e^{-r s}(C+f-d(s ; \tau)) d s+(1-\alpha) e^{-r \tau} V_{\tau}\right]
$$

where $(1-\alpha) V_{\tau}$ is the payment generated by the total debt at default. It follows that the actual cash flow to equity on time interval $[s, s+d s]$ is

$$
\left(\delta V_{s}-(1-\theta) C-f+d(s ; \tau)\right) d s
$$

yielding the equity value

$$
\mathbb{E}\left[\int_{0}^{\tau} e^{-r s}\left(\delta V_{s}-(1-\theta) C-f+d(s ; \tau)\right) d s\right]
$$

\footnotetext{
${ }^{5}$ Using an integration by parts, it is easy to show that the condition $F>\frac{\theta C}{r}$ implies the inequality (13) for any maturity profile $\mu$.

${ }^{6}$ See for instance Leland and Toft [22], Equations (7)-(10) or Hilberink and Rogers [14], Equations (2.7)$(2.10)$.
} 
Equation (17) is therefore the correct equity value at time 0 when the debt is rolled-over until the default time $\tau$. The literature however considers expression

$$
v(0 ; \tau)-D(0 ; \tau)
$$

to study the equity value. Thus the following questions: Is (18) a correct expression for the equity value? What is the link between the debt values $D(0 ; \tau)$ and $D_{\text {tot }}(0 ; \tau)$ ? How to formulate the equity holders' problem within a dynamic capital structure model with roll-over debt?

2. As we saw, the commitment to roll-over the debt according to a maturity profile $\mu$ yields a capital structure in which the total debt has a constant face value $F$ and pays a constant total coupon $C$. This capital structure is maintained until default because at any time the firm retires the amount $f d s$ and replaces it with a like amount of new debt. From this the literature argues that the asset value $V_{s}$ is a sufficient statistics for the newly issued debt. The overall conclusion is that corporate models with roll-over debt are time-homogenous meaning that the firm, debt and equity cash flows do not change as a function of $s$ but simply vary with $V_{s}$ and that, consequently, the optimal default policy must be characterized by a constant barrier strategy exactly as in the case of a debt with infinite maturity. Things are actually more complex than that. As a matter of fact consider a default policy $\tau_{a(.)}$ defined by the first time such that the firm's asset value crosses a deterministic time-dependent function $a(s)$. Clearly, the law of $\tau_{a(\text {.) }}$ given the available information at $s$ is time-dependent, so also will be the bond with coupon $c$ and face value 1 which price is given in (10) and in turn the instantaneous market value of newly issued bond $d\left(s ; \tau_{a(.)}\right)$. Without any restrictions ${ }^{7}$ on the default policy $\tau$, there is no reason to believe that the newly issued debt is time-independent. In models with roll-over debt, the debt and equity cash flows are not time-homogenous for any default policy $\tau$ as it is the case when debt is a consol bond. This in turn raises questions about the assumed optimality of a constant barrier strategy for the equity holders' problem.

3. The literature invokes the so called "smooth-pasting condition" for solving the equity holders' problem. The smooth pasting condition relies on stopping theory and reflects the $C^{1}$ nature of the value function associated with a stopping problem across the optimal boundary. When debt is a consol bond stopping problem (6) defines the equity value and the smooth-pasting condition can be correctly invoked. ${ }^{8}$ Things are different in a model with roll-over debt. Using Equations (12) and (14) together with the Ito's formula, expression (18) can be written as

$$
v(0 ; \tau)-D(0, \tau)=\mathbb{E}\left[\int_{0}^{\tau} e^{-r s} h\left(s, V_{s}\right) d s\right]
$$

\footnotetext{
${ }^{7}$ If the default policy is a constant barrier strategy then the cash flow to equity is time-independent.

${ }^{8}$ For a recent contribution on threshold strategies and smooth pasting condition, we refer to Dayanik and Karatzas [3] or to Villeneuve [27] whose Theorem 4.2 and Proposition 4.6 give simple conditions under which an optimal stopping time is given by a constant barrier strategy.
} 
where the time-dependent function $h(s, x)$ is defined by the relation

$$
h(s, x) \equiv x\left(\alpha \delta+(1-\alpha) \frac{1}{\bar{\Phi}(0)}(\delta \bar{\Phi}(s)+\Phi(s))\right)+C \theta-\frac{1}{\bar{\Phi}(0)}(C \bar{\Phi}(s)+F \Phi(s)) .
$$

All the previous articles restrict the study to constant barrier strategies $\tau_{B}=\inf \{s$ : $\left.V_{s} \leq B\right\}$ and use Equation (19) to derive closed form formula for the equity value. Under the assumption that the default policy is a constant barrier strategy the right hand side of (19) is a function of the current value of the firm's asset and the smoothpasting condition (7) posed in the literature can be written as

$$
\frac{\partial}{\partial V}\left(\mathbb{E}_{V}\left[\int_{0}^{\tau_{B}} e^{-r s} h\left(s, V_{s}\right) d s\right]\right)_{\mid V=B}=0
$$

Assuming that Equation (18) is a correct representation for the equity value (which again must be proven), is condition (21) really a smooth-pasting condition? What is the stopping problem associated with condition (21)? Is the equity holders' problem a standard stopping problem with solution characterized by the smooth-pasting principle?

Our clarification work goes through several steps that we develop in sections 3,4 and 5 . Section 3 presents the equity holders' problem in a model with roll-over debt. Section 4 confronts the existing literature with the equity holders' problem and answers to all the above questions. Section 5 discusses our results and investigates research directions for future work.

\section{Capital structure models with roll-over debt: The equity holders' problem.}

We show that the equity holders' problem involves a rational expectations equilibrium problem that requires to define and to characterize equilibrium default policies. We shall proceed as follows. First, we focus on the value process of equity in a model with roll-over debt in which equity holders do not take any strategic default decision. Thereby, we highlight a property of inter-temporal consistency that any model with roll-over debt must satisfy. Second, we introduce into the analysis the strategic behavior of equity holders and we develop our equilibrium concept. Third, we present the equity holders' problem. We shall see that it aims at selecting a payoff dominant equilibrium.

\subsection{Coherent default policies}

Let us consider an environment in which equity holders pre-commit at date 0 to roll over the debt and cannot take any strategic decision ex-post. The characteristics of the collection of debts that are continuously rolled over are the coupon rate $c$, the principal $f d t$, and the default policy of each newly issued debt. This yields a firm's default policy defined by a 
collection of stopping times $\hat{\tau}=\left(\hat{\tau}_{t}\right)_{t \geq 0}$ with $\hat{\tau}_{t} \in \mathcal{T}_{t, \infty}$. Thus, the cash flow to equity on time interval $[t, t+d t]$ is

$$
\left(\delta V_{t}-(1-\theta) C-f+d\left(t ; \hat{\tau}_{t}\right)\right) d t
$$

where $d\left(t ; \hat{\tau}_{t}\right)$ is the instantaneous market value of the newly issued debt at time $t \geq 0$ with default at time $\hat{\tau}_{t}$. What conditions must satisfy a default policy $\hat{\tau}=\left(\hat{\tau}_{t}\right)_{t \geq 0}$ ? Since all newly issued debts pay the same coupon rate $c$, have the same principal $f d t$ and the same maturity profile $\mu$, they must carry the same market price. Specifically, $d\left(t, \hat{\tau}_{s}\right)=$ $f \int_{0}^{\infty} b\left(t, t+u ; \hat{\tau}_{s}\right)[-d \Phi(u)]$, the instantaneous market price at date $t$ of the debt of generation $s$ (that is the debt issued at time $s<t$ with default at time $\hat{\tau}_{s}$ ) must coincide with $d\left(t, \hat{\tau}_{t}\right)=$ $f \int_{0}^{\infty} b\left(t, t+u ; \hat{\tau}_{t}\right)[-d \Phi(u)]$, the instantaneous market price at date $t$ of the debt of generation $t$ (that is the debt issued at time $t$ with default at time $\hat{\tau}_{t}$ ). This requires that $\hat{\tau}_{t}$ and $\hat{\tau}_{s}$ coincide on the set $\left\{\hat{\tau}_{s}>t\right\}$. This leads to the concept of coherent default policy:

Definition 1 We shall say that a default policy $\hat{\tau}=\left(\hat{\tau}_{t}\right)_{t \geq 0}$ is coherent if for every stopping time $S \leq T$ in $\mathcal{T}_{0, \infty}, \hat{\tau}_{S}=\hat{\tau}_{T}$ almost surely on the set $\left\{\hat{\tau}_{S} \geq T\right\}$.

Example 1 Let $\mathcal{D}$ be an open set of $(0, \infty)^{2}$ and let us consider the stopping time

$$
\tau_{\mathcal{D}}^{t}=\inf \left\{u \geq 0:\left(t+u, V_{t+u}\right) \notin \mathcal{D}\right\} \in \mathcal{T}_{0, \infty} .
$$

It results from the Strong Markov Property that the strategy $\tau^{\mathcal{D}}=\left(\tau_{t}^{\mathcal{D}}\right)_{t \geq 0}$ with

$$
\tau_{t}^{\mathcal{D}}=t+\tau_{\mathcal{D}}^{t} \circ \Theta_{t} \in \mathcal{T}_{t, \infty}
$$

is a coherent strategy. Observe that the constant barrier strategy strategies used in the literature are coherent. There are defined by relation (23) with $\mathcal{D}=(0, \infty) \times(B, \infty)$ and $B>0$.

The coherence property relies on the fact that each new debt is issued at market value. Although not formalized, the coherence property is fully recognized in the seminal paper of Leland [19]. ${ }^{9}$ The coherence property guarantees that a dynamic capital structure model with roll-over debt is coherent with a well functioning market. ${ }^{10}$ It implies that all issued debts must default simultaneously. Thus, the default time $\hat{\tau}_{0}$ of the debt of generation zero pins down the default policy of the firm. Consequently, the default policy $\hat{\tau}_{t}$ of the debt of generation $t$ satisfies $\hat{\tau}_{t}=\hat{\tau}_{0}$ on $\left\{\hat{\tau}_{0}>t\right\}$ and we can write $\left(E_{t}\right)_{t \geq 0}$, the value process of equity, under the form

$$
\begin{cases}E_{t}=\mathbb{E}\left[\int_{t}^{\hat{\tau}_{0}} e^{-r(s-t)}\left(\delta V_{s}-(1-\theta) C-f+d\left(s ; \hat{\tau}_{0}\right)\right) d s \mid \mathcal{F}_{t}\right] & \text { on }\left\{\hat{\tau}_{0}>t\right\}, \\ E_{t}=0 & \text { on }\left\{\hat{\tau}_{0} \leq t\right\} .\end{cases}
$$

We now turn to the strategic behavior of equity holders.

\footnotetext{
${ }^{9}$ See the last paragraph of page 9 and footnote 10 in Leland [19].

${ }^{10} \mathrm{In}$ absence of the coherence property an arbitrage opportunity at date $t$ would consist in selling (buying) the debt of generation $t$ and buying (selling) the debt of generation $s$.
} 


\subsection{Equilibrium default policies with commitment on the part of the firm.}

The timing of bankruptcy is determined by the decision of the firm (i.e equity holders) to cease rolling-over the debt. This strategic decision is taken after the roll-over debt being in place, that is after the default time $\hat{\tau}_{0}$ of the debt of generation 0 being announced. Intuitively, a default time $\hat{\tau}_{0}$ is an equilibrium default policy if and only if rational debt holders anticipate that default will indeed occur at $\hat{\tau}_{0}$. We develop our equilibrium concept under the assumption that the firm commit not to roll-over the debt beyond the announced date $\hat{\tau}_{0}$. In this setting the only strategic decision to be taken by equity holders is to decide whether or not to trigger bankruptcy before the announced default time $\hat{\tau}_{0}$. Rational debt holders anticipate that strategic behavior and accept to buy at every time newly issued debt only if they anticipate that equity holders will indeed roll over the debt until time $\hat{\tau}_{0}$. We prove below that the limited liability characterizes our equilibrium concept. We shall relax in section 4.2 our assumption of commitment on the part of the firm.

Thus, rational debt holders accept to buy the debt of generation 0 (issued in time interval $[0, d s])$ if and only if they anticipate that equity holders will never deviate from the announced default time $\hat{\tau}_{0}$. This will be the case if and only if the announced default time $\hat{\tau}_{0}$ is the smallest optimal stopping time solution to problem

$$
U_{t} \equiv \operatorname{ess} \sup _{\tau_{t} \in \mathcal{T}_{t, \infty}} \mathbb{E}\left[\int_{t}^{\tau_{t} \wedge \hat{\tau}_{0}} e^{-r(s-t)}\left(\delta V_{s}-(1-\theta) C-f+d\left(s ; \hat{\tau}_{0}\right)\right) d s \mid \mathcal{F}_{t}\right] \text {. }
$$

The upper bound $\tau_{t} \wedge \hat{\tau}_{0}$ in the integral reflects the commitment that debt is not rolled-over beyond time $\hat{\tau}_{0}$. Note that if there is an other stopping time $\underline{\tau}$ solution to problem (25) with $\mathbb{P}\left(\underline{\tau}<\hat{\tau}_{0}\right)>0$, then equity holders can profitably deviate by triggering default sooner at time $\underline{\tau}$ on the set $\left\{\underline{\tau}<\hat{\tau}_{0}\right\}$. This is the reason why we require $\hat{\tau}_{0}$ to be the smallest stopping time solution to (25). Therefore, for every $t$, an equilibrium default time $\hat{\tau}_{0}$ satisfies on $\left\{\hat{\tau}_{0}>t\right\}$ the equality $\hat{\tau}_{0}=\inf \left\{s \geq t, U_{s}=0\right\}$ a.s.

Definition 2 We shall say that a default time $\hat{\tau}_{0}$ is an equilibrium default policy if and only if $\hat{\tau}_{0}$ is the smallest optimal stopping time for (25).

Definition 2 implies that a default time $\hat{\tau}_{0}$ is an equilibrium default policy if and only if for every $t \geq 0 E_{t}=U_{t}$ almost surely where $E_{t}$ and $U_{t}$ are defined in (24) and (25). Thus, if $\hat{\tau}_{0}$ is an equilibrium default policy and $E_{t}=0$, then $\hat{\tau}_{0}=t$ almost surely. It is worth noting that any equilibrium default policy is clearly compatible with the limited liability condition that says that the value of equity remains non-negative at all times. Indeed, taking $\tau_{t}=t$ in (25) yields the value 0 from which we deduce that for any equilibrium default policy $\hat{\tau}_{0}$ the value of equity remains non-negative at all times as it must be. The next Proposition goes further and shows that the limited liability condition fully characterizes the equilibrium strategies.

Proposition $1 \mathrm{~A}$ default time $\hat{\tau}_{0}$ is an equilibrium default policy if and only if, for every $t \geq 0$, we have $E_{t} \geq 0$. 
Proof: We simply have to show the sufficient condition, that is, for every $t \geq 0, U_{t}=E_{t}$. Without loss of generality we write the proof for $t=0$. For any stopping time $\tau \in \mathcal{T}_{0, \infty}$, we have

$$
\begin{aligned}
E_{0} & =\mathbb{E}\left[\int_{0}^{\tau \wedge \hat{\tau}_{0}} e^{-r s}\left(\delta V_{s}-(1-\theta) C-f+d\left(s ; \hat{\tau}_{0}\right)\right) d s\right] \\
& +\mathbb{E}\left[\mathbb{E}\left[\int_{\tau \wedge \hat{\tau}_{0}}^{\hat{\tau}_{0}} e^{-r s}\left(\delta V_{s}-(1-\theta) C-f+d\left(s ; \hat{\tau}_{0}\right)\right) d s \mid \mathcal{F}_{\tau \wedge \hat{\tau}_{0}}\right]\right] \\
& =\mathbb{E}\left[\int_{0}^{\tau \wedge \hat{\tau}_{0}} e^{-r s}\left(\delta V_{s}-(1-\theta) C-f+d\left(s ; \hat{\tau}_{0}\right)\right) d s\right]+\mathbb{E}\left[e^{-r\left(\tau \wedge \hat{\tau}_{0}\right)} E_{\tau \wedge \hat{\tau}_{0}}\right] \\
& \geq \mathbb{E}\left[\int_{0}^{\tau \wedge \hat{\tau}_{0}} e^{-r s}\left(\delta V_{s}-(1-\theta) C-f+d\left(s ; \hat{\tau}_{0}\right)\right) d s\right]
\end{aligned}
$$

where the second equality comes from the Strong Markov property and the last inequality comes from the assumption that $E_{t}$ is positive on $[0, \infty)$ for every $t \geq 0$. Taking the supremum over all stopping times, we obtain that

$$
E_{0} \geq \sup _{\tau \in \mathcal{T}_{0, \infty}} \mathbb{E}\left[\int_{0}^{\tau \wedge \hat{\tau}_{0}} e^{-r s}\left(\delta V_{s}-(1-\theta) C-f+d\left(s ; \hat{\tau}_{0}\right)\right) d s\right]=U_{0}
$$

This ends the proof because the reverse inequality holds by definition.

In the previous literature the limited liability is often introduced as a necessary additional constraint to the equity holders' problem. The limited liability condition appears here naturally and is proven to characterize our equilibrium concept. The next section formulates the equity holders' problem.

\subsection{The equity holders' problem.}

We denote by $\mathcal{E} \subset \mathcal{T}_{0, \infty}$ the set of equilibrium default policies. We write the equity holders' problem as follows:

The equity holders' problem. In a dynamic capital structure model with roll-over debt equity holders select the default time $\tau$ in the set $\mathcal{E}$ in order to maximize at date 0 the value of equity. Formally, they solve the constrained optimal stopping problem

$$
\sup _{\tau \in \mathcal{E}} \mathbb{E}\left[\int_{0}^{\tau} e^{-r s}\left(\delta V_{s}-(1-\theta) C-f+d(s ; \tau)\right) d s\right] .
$$

We shall say that a solution to problem (26) is a payoff dominant equilibrium. ${ }^{11}$

Our formulation of the equity holders' problem deserves some comments.

\footnotetext{
${ }^{11}$ This vocabulary refers to Harsanyi and Selten [13].
} 
First, our formulation shows that the equity holders' problem is one of equilibrium selection. Describing the set $\mathcal{E}$ and ranking its elements is not an easy task. The question of existence and multiplicity of equilibria arises naturally. In the next section we study equilibrium policies that are hitting times of constant boundaries.

Second, our equilibrium concept and stopping problem (26) rationalize the key idea stated in Leland (1994) that the equity holders' objective is to maximize the present value of their claim while preserving the limited liability of equity. Indeed, a default time $\tau^{*}$ solution to problem (26) maximizes the present value of equity among default policies that yield a positive value of equity at any time before default.

Third, it is very intuitive that, at a stopping time solution to (26) the net cash flow rate to equity, $\delta V_{s}-(1-\theta) C-f+d(s ; \tau)$, must be negative (otherwise it would be optimal to continue the firm's activity at least for a short period of time). That is, at a stopping time solution to (26), net debt service requirements $((1-\theta) C-f)$ must exceed the available cash flow $\left(\delta V_{s}+d(s ; \tau)\right)$. As emphasized in the literature, the firm survives to this time because equity holders will issue new shares to absorb current losses. ${ }^{12}$ Default policies that generate a positive cash flow to equity at any time before default satisfy clearly the limited liability condition but are not solution to the equity holders' problem (26).

Finally, it is fair and important to say that Leland and Toft [22] have clearly in mind the question of default policies in a rational expectations equilibrium when they write page 992 "To determine the equilibrium bankruptcy-triggering asset value $V_{B}$ endogenously, we invoke the smooth pasting condition". However, Leland and Toft [22] as well as the rest of the literature did not define equilibrium default policies neither formulate the equity holders' problem nor justify representation (18) for the equity value. In addition, invoking the smooth pasting condition is questionable. To the best of our knowledge, its connection to the determination of a default policy in a rational expectations equilibrium has never been explained.

\section{Solving the equity holders' problem.}

We are now in a position to study the solution proposed in the literature and to answer the questions we raised in section 2. In section 4.1, we justify the representation (18) for the equity value used in the previous studies and we derive a new formulation for the equity holders' problem. In section 4.2, we study default policies defined by constant barrier strategies. We relax our assumption of equilibrium with commitment and we establish the existence and the uniqueness of equilibrium in barrier strategies which coincides with that derived in the literature. Thereby we provide a rationale for the seminal Leland [19] and Leland and Toft [22] papers.

\footnotetext{
${ }^{12}$ See for instance Leland and Toft [22] page 994.
} 


\subsection{An equivalent formulation for the equity holders' problem}

We prove below that the representation (18) of the equity value accounts for all the cash flow due to roll-over and thus coincides with expression (17) or equivalently that the value of all outstanding debt (12) and the total debt value (16) are the same. ${ }^{13}$ The underlying intuition is as follows: In corporate models with roll-over debt market frictions only come from interest tax shield and bankruptcy costs. It implies that the current value of the firm equals the current value of the firm without leverage plus the present value of the tax savings from debt, less the present value of bankruptcy costs (Equation (14)). In addition, the current value of the firm must agree with the sum of current values of equity and debt. The current value of equity equals the present value of the future cash flows to equity (Equation (17)). The current value of debt equals the present value of cash flows due to the existing debts (that is, due to rolling-over the debt up to the current time, Equation (12)). Thus, in a model with roll-over debt, the current value of the firm equals the sum of current values of equity and debt if and only if the value of all outstanding debt (12) equals the total debt value (16). The next Proposition, proven in Appendix A, confirms this intuition.

Proposition 2 For every stopping time $\tau \in \mathcal{T}_{0, \infty}$, we have $D(0, \tau)=D_{\text {tot }}(0, \tau)$.

Proposition 2 implies that the equity value (17) has also the representation (18) and that we have for every stopping time $\tau \in \mathcal{T}_{0, \infty}$

$$
\mathbb{E}\left[\int_{0}^{\tau} e^{-r s}\left(\delta V_{s}-(1-\theta) C-f+d(s ; \tau)\right) d s\right]=\mathbb{E}\left[\int_{0}^{\tau} e^{-r s} h\left(s, V_{s}\right) d s\right]
$$

where Equation (20) defines the function $h$. Therefore, the right hand side of (27) is a correct representation for the equity value. Interestingly, the function $h$ under the integral does not depend on the default policy $\tau$. This allows to derive closed form formula for the equity value once the maturity profile has been fixed. This is actually what the literature did assuming that $\tau$ is defined by a constant barrier strategy.

Thus, a consequence of Proposition 2 is that the equity holders' problem (26) can be written under the form

$$
\sup _{\tau \in \mathcal{E}} \mathbb{E}\left[\int_{0}^{\tau} e^{-r s} h\left(s, V_{s}\right) d s\right]
$$

A natural question is then to determine the stopping time solution to the unconstrained problem

$$
\sup _{\tau \in \mathcal{T}_{0, \infty}} \mathbb{E}\left[\int_{0}^{\tau} e^{-r s} h\left(s, V_{s}\right) d s\right]
$$

and to study whether or not it is an equilibrium default policy. We prove in Appendix B the following.

Proposition 3 There exists a non constant boundary $a^{*}($.$) defined on [0, \infty)$ such that $\tau_{a^{*}(.)}=\inf \left\{s: V_{s} \leq a^{*}(s)\right\}$ is an optimal stopping time for the problem

$$
\sup _{\tau \in \mathcal{T}_{0, \infty}} \mathbb{E}\left[\int_{0}^{\tau} e^{-r s} h\left(s, V_{s}\right) d s\right] .
$$

\footnotetext{
${ }^{13}$ We thank a referee for suggesting us this result.
} 
The coherent strategy $\left(t+\tau_{a^{*}(t+.)} \circ \Theta_{t}\right)_{t \geq 0}$ with $\tau_{a^{*}(t+.)}=\inf \left\{u \geq 0: V_{t+u} \leq a^{*}(t+u)\right\}$ does not belong to the set $\mathcal{E}$ of equilibrium default policies.

In words, optimal stopping time $\tau_{a^{*}(.)}$ would indeed maximize the value of equity right after the firm has issued its debt initially. However, debt holders anticipate that rolling over the debt until $\tau_{a^{*}(\text {.) }}$ will not remain optimal as the time goes by. That is $t+\tau_{a^{*}\left(t+\text {.)॰ } \Theta_{t}\right.}$ does not solve Problem (25). Consequently debt holders refuse to buy the newly issued debt on time interval $[0, d s]$ at price $d\left(0, \tau_{a^{*}(.)}\right)$ and thus the coherent policy $\left(t+\tau_{a^{*}(t+.)} \circ \Theta_{t}\right)_{t \geq 0}$ is not an equilibrium.

\subsection{Time-homogenous default policies.}

Let us characterize the constant barrier strategies $\tau_{B} \equiv \inf \left\{s \geq 0: V_{s} \leq B\right\}$ that are equilibrium default policies.

When the default policy is a hitting time $\tau_{B}$ of a constant barrier $B$, the market value of the newly issued debt in every time interval $(s, s+d s)$ only depends on the current value of the firm's asset. Using the Strong Markov Property we write Equation (24) under the form

$$
\begin{cases}E_{B}(x)=\mathbb{E}_{x}\left[\int_{0}^{\tau_{B}} e^{-r s}\left(\delta V_{s}-(1-\theta) C-f+d_{B}\left(V_{s}\right)\right) d s\right] & \text { if } x>B \\ E_{B}(x)=0 & \text { if } x \leq B\end{cases}
$$

where the mapping $x \longrightarrow d_{B}(x)$ follows from Equation (9) with $\tau=\tau_{B}$. That is,

$$
\begin{cases}d_{B}(x)=f \int_{0}^{\infty} \mathbb{E}_{x}\left[\int_{0}^{u \wedge \tau_{B}} c e^{-r v} d v+e^{-r u} \mathbb{1}_{\tau_{B}>u}\right][-d \Phi(u)] & \text { if } x>B \\ d_{B}(x)=\frac{f}{F}(1-\alpha) x & \text { if } x \leq B .\end{cases}
$$

The next corollary is a direct consequence of our previous results.

Corollary 1 The following assertions are equivalent.

(i) $\tau_{B}$ is an equilibrium default policy.

(ii) $\tau_{B}$ is an optimal stopping time for stopping problem

$$
\sup _{\tau \in \mathcal{T}_{0, \infty}} \mathbb{E}_{x}\left[\int_{0}^{\tau \wedge \tau_{B}} e^{-r s}\left(\delta V_{s}-(1-\theta) C-f+d_{B}\left(V_{s}\right)\right) d s\right]
$$

(iii) For every positive $x, E_{B}(x)=J_{B}(x)$ where $J_{B}$ is the value function associated with optimal stopping problem (33).

(iv) For every positive $x$,

$$
\mathbb{E}_{x}\left[\int_{0}^{\tau_{B}} e^{-r s}\left(\delta V_{s}-(1-\theta) C-f+d_{B}\left(V_{s}\right)\right) d s\right] \geq 0
$$


(v) For every positive $x, v_{B}(x)-D_{B}(x)=\mathbb{E}_{x}\left[\int_{0}^{\tau_{B}} e^{-r s} h\left(s, V_{s}\right) d s\right] \geq 0$ where $v_{B}$ and $D_{B}$ are obtained from Equations (11) and (14) with $\tau=\tau_{B}$.

We focus thereafter on constant barriers $B$ such that $B(1-\alpha) \leq F$. That is, the net value of the firm's assets in default is lower than the face value of debt. We shall also assume that $B_{\infty}(1-\alpha) \leq F$ where $B_{\infty}$ is the optimal default boundary when debt has an infinite maturity. ${ }^{14}$ The next proposition shows that the study of the right derivative of the function $E_{B}$ evaluated at $B$ leads to a simple characterization of equilibrium default policies $\tau_{B}$.

Proposition 4 For any maturity profile $\mu$, there is a unique threshold $B^{*}<\frac{F}{1-\alpha}$ such that $E_{B^{*}}$ is of class $C^{1}$. Moreover, for any $B \in\left[B^{*}, \frac{F}{1-\alpha}\right], \tau_{B}$ is an equilibrium default policy.

Proof: The proof relies on the following lemmas.

Lemma 1 Let us consider $B \leq \frac{F}{1-\alpha}$. The policy $\tau_{B}$ is an equilibrium default policy if and only if $E_{B}^{\prime}(B+)=\lim _{x \downarrow B} E_{B}^{\prime}(x)$ is nonnegative.

Proof of Lemma 1 The necessary condition is easy to establish. Indeed, if $E_{B}^{\prime}(B+)<$ 0 then the equality $E_{B}(B)=0$ implies that the equity-value $E_{B}$ is negative on a right neighborhood of $B$ and therefore (34) is not satisfied and $\tau_{B}$ cannot be an equilibrium default policy. Let us now assume that $E_{B}^{\prime}(B+) \geq 0$. It follows from Equation (31) that the function $E_{B}$ satisfies the ordinary differential equation

$$
\frac{\sigma^{2} x^{2}}{2} E_{B}^{\prime \prime}(x)+(r-\delta) x E_{B}^{\prime}(x)-r E_{B}(x)+g_{B}(x)=0 \text { for } x \geq B,
$$

with $E_{B}(x)=0$ for $x<B$ and where $g_{B}(x) \equiv \delta x-(1-\theta) C-f+d_{B}(x)$ is the cash flow to equity. We prove in Appendix $C$ that the cash flow to equity $g_{B}$ is increasing over $(0, \infty)$ for $B \leq \frac{F}{1-\alpha}$. We now show that $E_{B}^{\prime}(B+) \geq 0$ implies $E_{B}$ positive for every $x$. Assume the contrary, since $E_{B}$ is smooth for $x>B$ with $\lim _{x \uparrow \infty} E_{B}(x)>0$, the function $E_{B}$ can only be negative if there exists at least two real numbers $B<x_{0}<x_{1}$ such that $E_{B}\left(x_{0}\right)>0, E_{B}^{\prime}\left(x_{0}\right)=0, E_{B}^{\prime \prime}\left(x_{0}\right) \leq 0$ and $E_{B}\left(x_{1}\right)<0, E_{B}^{\prime}\left(x_{1}\right)=0, E_{B}^{\prime \prime}\left(x_{1}\right) \geq 0$. This in turn implies that $g_{B}\left(x_{0}\right) \geq 0$ and $g_{B}\left(x_{1}\right) \leq 0$ which contradicts the fact that $g_{B}$ is increasing. The function $E_{B}$ is thus positive, Equation (34) holds for every positive $x$ and therefore $\tau_{B}$ is an equilibrium default policy. This ends the proof of Lemma 1. Proposition 4 is then a consequence of the next lemma

Lemma 2 The following equality holds

$$
B E_{B}^{\prime}(B+)=k_{1}+k_{2} B
$$

where $k_{2}>0$ and $k_{1}<0$.

\footnotetext{
${ }^{14}$ Equation (7) defines the barrier $B_{\infty}$.
} 
We develop in Appendix $\mathrm{C}$ the proof of Lemma 2. It relies on equality

$$
E_{B}(x)=v_{B}(x)-D_{B}(x)=\mathbb{E}_{x}\left[\int_{0}^{\tau_{B}} e^{-r s} h\left(s, V_{s}\right) d s\right]
$$

and on standard properties of Geometric Brownian Motion. We also show in Appendix C that the inequality $(1-\alpha) B_{\infty}<F$ implies that $B^{*} \equiv \frac{-k_{1}}{k_{2}}<\frac{F}{1-\alpha}$. Then, it follows from Lemma 1 and Lemma 2 that $\tau_{B}$ with $B \in\left[B^{*}, \frac{F}{1-\alpha}\right]$ is an equilibrium default policy and that $E_{B^{*}}$ is of class $C^{1}$.

Note that, from the proof of Lemma $2, B^{*}$ is the unique real number that satisfies the smooth pasting condition (21) and corresponds to the default threshold considered in the literature. We fully explain the role of the smooth pasting condition (21) in the next paragraphs where we relax our assumption of equilibrium with commitment.

Let us assume that the market (that is debt holders) anticipate that the firm will use hitting times of constant barriers. Under these market's anticipations, we relax our assumption of commitment on the part of the firm and establish that the policy $\tau_{B^{*}}$ sustains the unique rational expectations equilibrium in barrier strategies. To relax the assumption of commitment and to take into account equity holders' deviation after the announced date of default we need to specify off-equilibrium debt prices for any time $s>\hat{\tau}_{0}$. That is we must define $d\left(s ; \hat{\tau}_{0}\right)$ for $s>\hat{\tau}_{0}$. When debt holders anticipate that the firm will use hitting times of constant barriers, a consistent way of formalizing off-equilibrium prices is to assume that debt holders believe that default is imminent whenever the current value of the firm's asset is below the announced barrier $B$. Thus, under that assumption, the market value of each new debt when $V_{s} \leq B$ is $\frac{f}{F}(1-\alpha) V_{s}$. Therefore, off-equilibrium debt prices are time-independent and are defined by Equation (32). This leads us to restate the definition of an equilibrium as follows

Definition 3 We shall say that a stopping time $\tau_{B} \equiv \inf \left\{s \geq 0: V_{s} \leq B\right\}$ is an equilibrium default policy if and only if $\tau_{B}$ is an optimal stopping time for problem

$$
\hat{J}_{B}(x)=\sup _{\tau \in \mathcal{T}_{0, \infty}} \mathbb{E}_{x}\left[\int_{0}^{\tau} e^{-r s}\left(\delta V_{s}-(1-\theta) C-f+d_{B}\left(V_{s}\right)\right) d s\right] .
$$

We obtain:

Proposition 5 According to Definition 3, the policy $\tau_{B^{*}}$ is the unique equilibrium default policy in constant barrier strategies and we have $E_{B^{*}}(x)=\hat{J}_{B^{*}}(x)$ for all $x \geq 0$.

Proof: According to Theorem 4.2 in [27], a threshold strategy $\hat{B}(B)$ is optimal for Problem (36), the function $\hat{J}_{B}$ is of class $C^{1}$ and can be written in terms of the free boundary problem

$$
\left\{\begin{array}{l}
\frac{\sigma^{2} x^{2}}{\hat{J}^{2}} \hat{J}_{B}^{\prime \prime}(x)+(r-\delta) x \hat{J}_{B}^{\prime}(x)-r \hat{J}_{B}(x)+g_{B}(x)=0, \quad x \geq \hat{B}(B) \\
\hat{J}_{B}(x)=0, \quad 0 \leq x \leq \hat{B}(B) \\
\hat{J}_{B}^{\prime}(\hat{B}(B))=0
\end{array}\right.
$$


However, we know from Proposition 4 that the pair $\left(E_{B^{*}}, B^{*}\right)$ is also a $C^{1}$ solution to the free boundary problem (37) written with $B=B^{*}$. This implies that $\hat{J}_{B^{*}}=E_{B^{*}}$ and $\hat{B}\left(B^{*}\right)=B^{*}$. It also implies that $B^{*}$ is the unique fixed point of the mapping $\hat{B}$. Indeed any other fixed point $B^{* *} \neq B^{*}$ would satisfy $\hat{J}_{B^{* *}}=E_{B^{* *}}$ which contradicts the fact that $B^{*}$ is the unique threshold such that $E_{B^{*}}$ is of class $C^{1}$. It follows that $\tau_{B^{*}}$ is the unique equilibrium according to Definition 3.

We knew from section 3 that limited liability characterizes equilibrium default policies with commitment. Defining off-equilibrium beliefs allows us to pin down the lowest constant barrier compatible with limited liability as the unique equilibrium default policy. It follows that $\tau_{B^{*}}$ sustains the unique rational expectations equilibrium in barrier strategies. Specifically, if the market anticipates that the firm will use the default policy $\tau_{B^{*}}$, then it is optimal for the firm to use the policy $\tau_{B^{*}}$ given that it could have use any other policy. Therefore, the firm fulfills the market's anticipation and Proposition 5, which holds for any maturity profile, provides a rationale for the Leland [19] and Leland and Toft [22] papers.

\section{Discussion}

In this section, we discuss our results and address challenging questions for future work.

Geometric Brownian motion. Up to section 4.2 our study does not rely on the assumption that the state variable follows a Geometric Brownian Motion. This is no more the case for Proposition 4 and Proposition 5 that crucially rely on the property of the density function of the first passage time of a Geometric Brownian Motion to a barrier $B$. In particular, it is unlikely that $B E_{B}^{\prime}\left(B_{+}\right)$being a linear function of $B$ for any diffusion process. Clearly it will be interesting to study the connection between default policy in a rational expectations equilibrium and optimal stopping theory when the state variable follows more general Lévy processes. Such a study will draw on Hilberink and Rogers [14], Kyprianou and Surya [16] and Chen and Kou [2] who have already introduced Lévy processes in capital structure models with roll-over debt.

Bankruptcy allocation. Following the literature, we have assumed that, in default, debt holders receive all assets remaining after paying bankruptcy costs. Assume now that equity holders get at bankruptcy $\max \left((1-\alpha) V_{\tau}-F, 0\right)$ (and debt holders $\min \left((1-\alpha) V_{\tau}, F\right)$ ). How do these payoffs impact our results? Proceeding analogously as in the proof of Proposition 1 , we obtain the following characterization.

Proposition 6 A constant barrier strategy $\tau_{B}$ is an equilibrium default policy if and only if, for every $x \geq 0$, we have $E_{B}(x) \geq((1-\alpha) x-F)_{+}$.

Whether the constant barrier strategy $\tau_{B^{*}}$ remains an equilibrium default policy according to Definition 2 depends on the value of the parameters of the model. For instance, in the 
framework of the exponential model ${ }^{15}$, we get

$$
\begin{aligned}
E_{B^{*}}(x) & \geq \mathbb{E}_{x}\left[\int_{0}^{\infty} e^{-r s}\left(\delta V_{s}-(1-\theta) C-m F+d_{B^{*}}\left(V_{s}\right) d s\right]\right. \\
& \geq x+\frac{\theta C}{r}-\frac{C+m F}{r} \\
& >x-F
\end{aligned}
$$

for $F \geq(1-\theta) \frac{C}{r}$ and $m$ small enough. Therefore, within this set of parameters, $E_{B^{*}}(x)$ dominates $((1-\alpha) x-F)_{+}$for every $x \geq 0$ and $\tau_{B^{*}}$ is still an equilibrium default policy. On the contrary, the policy $\tau_{B^{*}}$ is no more an equilibrium default policy if $F<(1-\theta) \frac{C}{r}$, $\alpha=0$ and $m$ sufficiently small. Indeed, $\alpha=0$ implies $^{16} E_{B^{*}}(x) \underset{\infty}{\sim} x+\frac{\theta C}{r}-\frac{C+m F}{r+m}$ and $F<(1-\theta) \frac{C}{r}$ yields $F<\frac{C+m F}{r+m}-\frac{\theta C}{r}$ for $m$ small. It follows that $E_{B^{*}}(x)$ cannot dominate $(x-F)_{+}$for $x$ large which means that $\tau_{B^{*}}$ is not an equilibrium default policy. Thus, depending on the parameter values, the bankruptcy allocation can have a dramatic impact on the equilibrium outcomes and remains an important issue for future work.

Beyond time-homogenous default policies. We show below that, when the firm loses all its value at bankruptcy, the barrier strategy $\tau_{B^{*}}$ is a payoff dominant equilibrium. That is, according to (26), for any $\tau^{*} \in \mathcal{E}$ and for any current value $x$ of the firm's asset we have

$$
E\left(0 ; \tau^{*}\right)=\mathbb{E}\left[\int_{0}^{\tau^{*}} e^{-r s}\left(\delta V_{s}-(1-\theta) C-f+d\left(s ; \tau^{*}\right)\right) d s\right] \leq E_{B^{*}}(x) .
$$

It follows that, when the firm loses all its value at bankruptcy, the equity holder's problem admits a unique equilibrium which coincides with that derived in the literature.

The reason why bankruptcy costs play a central role is as follows. In corporate models where debt is not rolled-over equity holders do not care about bankruptcy costs $\alpha$, which are borne at default by debt holders and the optimal stopping rule solution to the equity holders' problem is invariant to $\alpha$. In corporate models with roll-over debt equity holders care about bankruptcy costs because each newly issued debt is issued at market value. Other things equal, the equity cash flow increases with the market value of the newly issued debt. When there is no recovery at default, an increase of the length of time before bankruptcy always increases the market value of the newly issued debt and consequently also increases the equity-cash flow. We prove that this monotonicity property implies that the constant barrier strategy $\tau_{B^{*}}$ is the unique solution to the equity holders' problem (26), precisely:

Proposition 7 If there is no recovery at default, for any current value $x$ of the firm's asset, any stopping time $\tau^{*}$ solution to (26) satisfies $\tau^{*}=\tau_{B^{*}} \mathbb{Q}_{x}$-a.s.

Proof: Suppose there is no recovery at default, that is, $\alpha=1$. A direct computation shows

\footnotetext{
${ }^{15}$ In the exponential model, $\mu(d s)=m e^{-m s} d s$ with $m>0$ and $\Phi(s)=m \bar{\Phi}(s)=e^{-m s}$ which yields the relation $f=m F$

${ }^{16}$ The computation is straightforward. See for instance Leland [19] for explicit formula in the exponential model.
} 
that the instantaneous market value of a newly issued debt $d(s ; \tau)$ satisfies the monotonicity property: if $\tau \leq \tilde{\tau}$ then $d(s ; \tau) \leq d(s ; \tilde{\tau})$. In words, the larger the length of time before default, the larger the market debt value issued at $s$ is. Let us consider an equilibrium default policy $\tau^{*}$. Using the mapping $\hat{B}$ and the monotonicity property, we prove below that $\tau^{*} \leq \tau_{B^{*}} \mathbb{Q}_{x^{-}}$a.s. This latter inequality and again the monotonicity property will imply Proposition 7 .

Let us consider $\hat{J}_{0}$, the value function associated with optimal stopping problem (36) for the boundary $B=0$. That is,

$$
\hat{J}_{0}(x)=\sup _{\tau \in \mathcal{T}_{0, \infty}} \mathbb{E}_{x}\left[\int_{0}^{\tau} e^{-r s}\left(\delta V_{s}-(1-\theta) C-f+d_{0}\right) d s\right]
$$

where, with a slight abuse of notation, $d_{0}$ denotes the market value of the roll-over riskless debt. ${ }^{17}$ Let us denote by $\tau_{\hat{B}(0)}$ an optimal stopping time for the above problem and let us show that $\tau^{*} \leq \tau_{\hat{B}(0)} \mathbb{Q}_{x}$-a.s. Suppose by way of contradiction that $\mathbb{Q}_{x}\left(\tau^{*}>\tau_{\hat{B}(0)}\right)>0$. On the set $\left\{\tau^{*}>\tau_{\hat{B}(0)}\right\}$, Proposition 1 implies that

$$
E_{\tau_{\hat{B}(0)}}=\mathbb{E}\left[\int_{\tau_{\hat{B}(0)}}^{\tau^{*}} e^{-r\left(s-\tau_{\hat{B}(0)}\right)}\left(\delta V_{s}-(1-\theta) C-f+d\left(s ; \tau^{*}\right)\right) d s \mid \mathcal{F}_{\tau_{\hat{B}(0)}}\right]>0 .
$$

On the other hand the monotonicity property yields

$$
\begin{aligned}
& E_{\tau_{\hat{B}(0)}} \leq \mathbb{E}\left[\int_{\tau_{\hat{B}(0)}}^{\tau^{*}} e^{-r\left(s-\tau_{\hat{B}(0)}\right)}\left(\delta V_{s}-(1-\theta) C-f+d_{0}\right) d s \mid \mathcal{F}_{\tau_{\hat{B}(0)}}\right]
\end{aligned}
$$

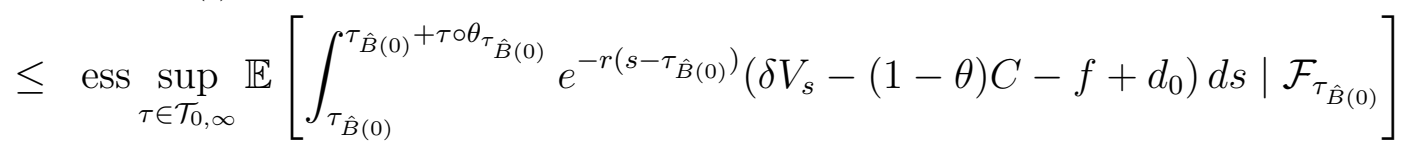

$$
\begin{aligned}
& =\hat{J}_{0}(\hat{B}(0)) \\
& =0
\end{aligned}
$$

where (39) comes from the Strong Markov Property. Inequalities (38) and (40) contradict inequality $\mathbb{Q}_{x}\left(\tau^{*}>\tau_{\hat{B}(0)}\right)>0$, thus $\tau^{*} \leq \tau_{\hat{B}(0)} \mathbb{Q}_{x}$-a.s. Proceeding recursively, we construct an increasing sequence $\left(b_{n}\right)_{n \geq 0}$ with $b_{0}=\hat{B}(0)$ such that $b_{n+1}=\hat{B}\left(b_{n}\right)$ and $\tau^{*} \leq \tau_{b_{n}} \mathbb{Q}_{x}$-a.s. for any $n$. Two cases have to be considered. Either there exists $n$ such that $b_{n} \geq B^{*}$, or for every $n$ we have $b_{n} \leq B^{*}$. The first case clearly yields the result. In the second case the sequence $\left(b_{n}\right)_{n \geq 0}$ is increasing and bounded and converges to $B^{*}$, the fixed point of the mapping $\hat{B}$. This again yields the result. Thus, in any case we have that $\tau^{*} \leq \tau_{B^{*}} \mathbb{Q}_{x^{-}}$-a.s. Now, using for the last time the monotonicity property one gets

$$
\begin{aligned}
E\left(0 ; \tau^{*}\right) & \leq \mathbb{E}_{x}\left[\int_{0}^{\tau^{*}} e^{-r s}\left(\delta V_{s}-(1-\theta) C-f+d_{B^{*}}\left(V_{s}\right)\right) d s\right] \\
& \leq \hat{J}_{B^{*}}(x) \\
& =E_{B^{*}}(x) .
\end{aligned}
$$

${ }^{17}$ We have $d_{0}=f \int_{0}^{\infty}\left(\frac{c}{r}\left(1-e^{-r u}\right)+e^{-r u}\right)[-d \Phi(u)]$. 
which proves that any stopping time $\tau^{*}$ solution to $(26)$ satisfies $\tau^{*}=\tau_{B^{*}} \mathbb{Q}_{x^{-}}$a.s.

The fact that $\tau_{B^{*}}$ is a payoff dominant equilibrium relies on the monotonicity property of the equity cash flow. An easy computation shows that, when there is a strictly positive recovery at default, postponing default and paying to debt holders the payment flow $c d t$ for some additional period of time does not necessarily increase the value of the newly issued debt. The monotonicity property of the equity cash flow does not hold anymore and, unfortunately, we lose our argument for proving that $\tau_{B^{*}}$ is solution to (26). In that case there are actually no clear reasons why $\tau_{B^{*}}$ should be again a payoff dominant equilibrium policy and, to the best of our knowledge, solving the equity holders' problem (26) still remains an open question.

\section{Conclusion.}

Roll-over debt structure models are much more involved than what academics were thinking and require to solve a difficult rational expectations equilibrium problem. This paper studies this equilibrium problem. Beyond the various results proven in the paper, we summarize our message as follows. If the market anticipates that the firm will use default policies defined by constant barrier strategies and believes that default is imminent whenever the current level of firm's asset is below the announced default barrier, then there exists a unique equilibrium in constant barrier strategies which coincides with that derived in the literature. Solving the equity-holders' problem under more general hypothesis remains a challenging question. 


\section{Appendix A}

Appendix A develops the proof of Proposition 2. Proceeding analogously as in Equation (12), we write $d(s ; \tau)$ under the form:

$$
\begin{aligned}
d(s ; \tau) & =f c \mathbb{E}\left[\int_{s}^{\tau} e^{-r(u-s)} \Phi(u-s) d u \mid \mathcal{F}_{s}\right]+f \mathbb{E}\left[\int_{s}^{\tau} e^{-r(u-s)}(-d \Phi(u-s)) d u \mid \mathcal{F}_{s}\right] \\
& +\frac{(1-\alpha) f}{F} \mathbb{E}\left[e^{-r(\tau-s)} V_{\tau} \Phi(\tau-s) \mid \mathcal{F}_{s}\right] .
\end{aligned}
$$

We then develop Equation (16) as follows:

$$
\begin{aligned}
D_{t o t}(0 ; \tau) & =\mathbb{E}\left[\int_{0}^{\tau} e^{-r s}(C+f) d s\right]-c f \mathbb{E}\left[\int_{0}^{\tau} e^{-r s} \mathbb{E}\left[\int_{s}^{\tau} e^{-r(u-s)} \Phi(u-s) d u \mid \mathcal{F}_{s}\right] d s\right] \\
& -f \mathbb{E}\left[\int_{0}^{\tau} e^{-r s} \mathbb{E}\left[\int_{s}^{\tau} e^{-r(u-s)}(-d \Phi(u-s)) d u \mid \mathcal{F}_{s}\right] d s\right] \\
& -\frac{(1-\alpha) f}{F} \mathbb{E}\left[\int_{0}^{\tau} e^{-r s} \mathbb{E}\left[e^{-r(\tau-s)} V_{\tau} \Phi(\tau-s) \mid \mathcal{F}_{s}\right] d s\right]+(1-\alpha) \mathbb{E}\left(e^{-r \tau} V_{\tau}\right) \\
& =A_{1}+A_{2}+A_{3}+A_{4}+A_{5} .
\end{aligned}
$$

Fubini-Tonelli Theorem yields

$$
\begin{aligned}
A_{4} & =-\frac{(1-\alpha) f}{F} \mathbb{E}\left[\int_{0}^{\tau} e^{-r \tau} V_{\tau} \Phi(\tau-s) d s\right] \\
& =-\frac{(1-\alpha) f}{F} \mathbb{E}\left[e^{-r \tau} V_{\tau} \int_{0}^{\tau} \Phi(u) d u\right] \\
& =-\frac{(1-\alpha) f}{F} \mathbb{E}\left[e^{-r \tau} V_{\tau}(\bar{\Phi}(0)-\bar{\Phi}(\tau))\right] .
\end{aligned}
$$

Because $F=f \bar{\Phi}(0)$, we get

$$
\left.A_{4}+A_{5}=(1-\alpha) \mathbb{E}\left[e^{-r \tau} V_{\tau} \frac{\bar{\Phi}(\tau)}{\bar{\Phi}(0)}\right)\right]
$$

We also have that,

$$
\begin{aligned}
A_{2} & =-c f \mathbb{E}\left[\int_{0}^{\tau} \int_{s}^{\tau} e^{-r u} \Phi(u-s) d u d s\right] \\
& =-c f \mathbb{E}\left[\int_{0}^{\tau} e^{-r u} \int_{0}^{u} \Phi(u-s) d s d u\right] \\
& =-c f \mathbb{E}\left[\int_{0}^{\tau} e^{-r u} \int_{0}^{u} \Phi(t) d t d u\right] \\
& =-c f \mathbb{E}\left[\int_{0}^{\tau} e^{-r u}(\bar{\Phi}(0)-\bar{\Phi}(u)) d u\right] .
\end{aligned}
$$


In the same manner,

$$
\begin{aligned}
A_{3} & =-f \mathbb{E}\left[\int_{0}^{\tau} e^{-r u} \int_{0}^{u}(-d \Phi(u-s)) d u\right] \\
& =-f \mathbb{E}\left[\int_{0}^{\tau} e^{-r u}(1-\Phi(u)) d u\right] .
\end{aligned}
$$

Because $C=c F$, we get

$$
\begin{aligned}
A_{1}+A_{2}+A_{3} & =\mathbb{E}\left[\int_{0}^{\tau} e^{-r s}(C+f-c f(\bar{\Phi}(0)-\bar{\Phi}(s))-f(1-\Phi(s))) d s\right] \\
& =\mathbb{E}\left[\int_{0}^{\tau} e^{-r s}\left(\frac{C \bar{\Phi}(s)+F \Phi(s)}{\bar{\Phi}(0)}\right) d s\right]
\end{aligned}
$$

from which, we deduce the result.

\section{Appendix B}

Appendix B is devoted to the proof of Proposition 3. We first prove that for every $x>0$ and $t>0$,

$$
\mathbb{E}_{t, x}\left[\int_{0}^{\infty} e^{-r s}\left|h\left(t+s, V_{t+s}\right)\right| d s\right]<+\infty .
$$

Relation (20) gives $|h(t+s, x)| \leq K_{0} x+K_{1}$ where $K_{0}, K_{1}$ are two positive constants. Therefore, it suffices to prove that $\int_{0}^{\infty} e^{-r s} V_{t+s} d s$ is integrable under $\mathbb{Q}_{t, x}$. This comes from Fubini-Tonelli Theorem which yields

$$
\begin{aligned}
\mathbb{E}_{t, x}\left[\int_{0}^{\infty} e^{-r s} V_{t+s} d s\right] & =\int_{0}^{\infty} \mathbb{E}_{t, x}\left(e^{-r s} V_{t+s}\right) d s \\
& =\int_{0}^{\infty} x e^{-\delta s} d s \\
& =\frac{x}{\delta} .
\end{aligned}
$$

Let us denote

$$
H(t, x)=\mathbb{E}_{t, x}\left[\int_{0}^{\infty} e^{-r s} h\left(t+s, V_{t+s}\right) d s\right]
$$


Assuming that $V_{0}=x$, we deduce from Equations (12) and (14) that

$$
\begin{aligned}
v(0 ; \tau)-D(0 ; \tau) & =\mathbb{E}_{x}\left[\int_{0}^{\tau} e^{-r s} h\left(s, V_{s}\right) d s\right] \\
& =H(0, x)-\mathbb{E}_{x}\left[\int_{\tau}^{\infty} e^{-r s} h\left(s, V_{s}\right) d s\right] \\
& =H(0, x)-\mathbb{E}_{x}\left[\mathbb{E}\left[\int_{\tau}^{\infty} e^{-r s} h\left(s, V_{s}\right) d s \mid \mathcal{F}_{\tau}\right]\right] \\
& =H(0, x)-\mathbb{E}_{x}\left[e^{-r \tau} H\left(\tau, V_{\tau}\right)\right] \\
& =x-\int_{0}^{\infty} e^{-r s}\left(C \frac{\bar{\Phi}(s)}{\bar{\Phi}(0)}+F \frac{\Phi(s)}{\bar{\Phi}(0)}-\theta C\right) d s \\
& +\mathbb{E}_{x}\left[e^{-r \tau}\left(\beta(\tau)-\gamma(\tau) V_{\tau}\right)\right]
\end{aligned}
$$

with

$$
\beta(t)=\int_{0}^{\infty} e^{-r s}\left(C \frac{\bar{\Phi}(t+s)}{\bar{\Phi}(0)}+F \frac{\Phi(t+s)}{\bar{\Phi}(0)}-\theta C\right) d s
$$

and

$$
\gamma(t)=\alpha+(1-\alpha) \frac{\bar{\Phi}(t)}{\bar{\Phi}(0)} .
$$

Note that the functions $\beta$ and $\gamma$ are bounded. Therefore,

$$
\begin{aligned}
\sup _{\tau \in \mathcal{T}_{0, \infty}}(v(0 ; \tau)-D(0 ; \tau)) & =x-\int_{0}^{\infty} e^{-r s}\left(C \frac{\bar{\Phi}(s)}{\bar{\Phi}(0)}+F \frac{\Phi(s)}{\bar{\Phi}(0)}-\theta C\right) d s \\
& +\sup _{\tau \in \mathcal{T}_{0, \infty}} \mathbb{E}_{x}\left[e^{-r \tau}\left(\beta(\tau)-\gamma(\tau) V_{\tau}\right)\right] .
\end{aligned}
$$

To prove Proposition 3 we have to study the stopping problem

$$
P(x) \equiv \sup _{\tau \in \mathcal{T}_{0, \infty}} \mathbb{E}_{x}\left[e^{-r \tau}\left(\beta(\tau)-\gamma(\tau) V_{\tau}\right)\right],
$$

with the convention that $\beta(\tau)-\gamma(\tau) V_{\tau}=0$ on $\{\tau=\infty\}$. Four lemmas drive the proof.

Lemma 3 Let $\mathcal{P}:[0, \infty) \times(0, \infty) \longrightarrow \mathbb{R}$ be the value function associated with the Markov bi-dimensional optimal stopping problem in the $(t, x)$ space

$$
\mathcal{P}(t, x)=\sup _{\tau \in \mathcal{T}_{0, \infty}} \mathbb{E}_{x}\left[e^{-r \tau} g\left(t+\tau, V_{\tau}\right)\right],
$$

where $g(t, u) \equiv \beta(t)-\gamma(t) u$. Then the value function $P$ associated with stopping problem (41) satisfies

$$
P(x)=\mathcal{P}(0, x) .
$$

Furthermore, the stopping time

$$
\tau^{*} \equiv \inf \left\{s \geq 0 \mid \mathcal{P}\left(t+s, V_{s}\right)=g\left(t+s, V_{s}\right)\right\}
$$

is optimal for problem (42). 
Proof of Lemma 3 We follow the optimal stopping theory (see [15]) and consider the Snell envelope process

$$
\left(\mathcal{P}_{t}\right)_{t \geq 0} \equiv\left(\operatorname{ess} \sup _{\tau \in \mathcal{T}_{t, \infty}} \mathbb{E}\left[e^{-r \tau} g\left(\tau, V_{\tau}\right) \mid \mathcal{F}_{t}\right]\right)_{t \geq 0} .
$$

Because $\beta$ is bounded, we have $\sup _{t \geq 0} \mathbb{E}\left[e^{-r t} \mathcal{P}_{t}\right]<+\infty$. Thus, $\mathcal{P}_{t}$ is the smallest supermartingale that dominates $\left(e^{-r t} g\left(t, V_{t}\right)\right)_{t \geq 0}$. We have that

$$
\begin{aligned}
\mathcal{P}_{t} & =e^{-r t} \operatorname{ess} \sup _{\tau \in \mathcal{T}_{0, \infty}} \mathbb{E}\left[e^{-r \tau} g\left(t+\tau, V_{t+\tau}\right) \mid \mathcal{F}_{t}\right] \\
& =e^{-r t} \sup _{\tau \in \mathcal{T}_{0, \infty}} \mathbb{E}_{V_{t}}\left[e^{-r \tau} g\left(t+\tau, V_{\tau}\right)\right] \\
& =e^{-r t} \mathcal{P}\left(t, V_{t}\right)
\end{aligned}
$$

where the second equality comes from the Markov property. It therefore results that, as announced,

$$
P(x) \equiv \sup _{\tau \in \mathcal{T}_{0, \infty}} \mathbb{E}_{x}\left[e^{-r \tau} g\left(\tau, V_{\tau}\right)\right]=\mathcal{P}(0, x) .
$$

Moreover, according to the optimal stopping theory, the process $\left(\mathcal{P}_{u \wedge \tau_{t}^{*}}\right)_{u \geq t}$ is an $\left(\mathcal{F}_{u}\right)$-martingale with

$$
\tau_{t}^{*} \equiv \inf \left\{u \geq t \mid \mathcal{P}_{u}=e^{-r u} g\left(u, V_{u}\right)\right\} .
$$

Therefore, Optional Sampling Theorem gives for every integer $n$,

$$
\begin{aligned}
\mathcal{P}_{t} & =\mathbb{E}\left[\mathcal{P}_{\tau_{t}^{*}} \mathbb{1}_{\left\{\tau_{t}^{*}<n\right\}} \mid \mathcal{F}_{t}\right]+\mathbb{E}\left[\mathcal{P}_{n} \mathbb{1}_{\left\{\tau_{t}^{*} \geq n\right\}} \mid \mathcal{F}_{t}\right] \\
& \leq \mathbb{E}\left[e^{-r \tau_{t}^{*}} g\left(\tau_{t}^{*}, V_{\tau_{t}^{*}}\right) \mathbb{1}_{\left\{\tau_{t}^{*}<n\right\}} \mid \mathcal{F}_{t}\right]+e^{-r n} \int_{0}^{\infty} e^{-r u}\left(C \frac{\bar{\Phi}(u)}{\bar{\Phi}(0)}+F \frac{\Phi(u)}{\bar{\Phi}(0)}\right) d u
\end{aligned}
$$

where the last inequality results from the relation

$$
\begin{aligned}
\mathbb{E}\left[\mathcal{P}_{n} \mathbb{1}_{\left\{\tau_{t}^{*} \geq n\right\}} \mid \mathcal{F}_{t}\right] & \leq e^{-r n} \mathbb{E}\left[\beta(n) \mathbb{1}_{\left\{\tau_{t}^{*} \geq n\right\}} \mid \mathcal{F}_{t}\right] \\
& \leq e^{-r n} \beta(n) \\
& \leq e^{-r n} \int_{0}^{\infty} e^{-r u}\left(C \frac{\bar{\Phi}(u)}{\bar{\Phi}(0)}+F \frac{\Phi(u)}{\bar{\Phi}(0)}\right) d u .
\end{aligned}
$$

Letting $n$ tend to infinity in (45), we obtain

$$
\mathcal{P}_{t} \leq \mathbb{E}\left[e^{-r \tau_{t}^{*}} g\left(\tau_{t}^{*}, V_{\tau_{t}^{*}}\right) \mathbb{1}_{\left\{\tau_{t}^{*}<\infty\right\}} \mid \mathcal{F}_{t}\right] .
$$

Using the convention on the set $\left\{\tau_{t}^{*}=\infty\right\}$, we have

$$
\mathcal{P}_{t} \leq \mathbb{E}\left[e^{-r \tau_{t}^{*}} g\left(\tau_{t}^{*}, V_{\tau_{t}^{*}}\right) \mid \mathcal{F}_{t}\right]
$$

Because the reverse inequality always holds, we get

$$
\mathcal{P}_{t}=\mathbb{E}\left[e^{-r \tau_{t}^{*}} g\left(\tau_{t}^{*}, V_{\tau_{t}^{*}}\right) \mid \mathcal{F}_{t}\right] .
$$


Using the Markov Property and the equality $\mathcal{P}_{t}=e^{-r t} \mathcal{P}\left(t, V_{t}\right)$, we get

$$
\begin{aligned}
\mathcal{P}\left(t, V_{t}\right) & =\mathbb{E}\left[e^{-r\left(\tau_{t}^{*}-t\right)} g\left(\tau_{t}^{*}, V_{\tau_{t}^{*}}\right) \mid \mathcal{F}_{t}\right] \\
& =\mathbb{E}_{V_{t}}\left[e^{-r \tau^{*}} g\left(t+\tau^{*}, V_{\tau^{*}}\right)\right]
\end{aligned}
$$

with $\tau^{*}$ defined by Equation (43). This ends the proof of Lemma 2.

Let us now consider the stopping region of problem (42):

$$
S \equiv\left\{( t , x ) \in \left[0, \infty\left[^{2} \mid \mathcal{P}(t, x)=g(t, x)\right\}\right.\right.
$$

and its t-sections

$$
S_{t} \equiv\{V \in[0, \infty[\mid \mathcal{P}(t, x)=g(t, x)\} .
$$

Remark that the stopping region $S$ can be written as $S=\cup_{t \geq 0}\{t\} \times S_{t}$ and that the optimal stopping time $\tau^{*}$ is the first time when the process $\left(t, V_{t}\right)$ hits the stopping region $S$. We show the following.

Lemma 4 The t-sections $S_{t}$ are left-connected. That is, there is a function $a^{*}$ defined on $(0, \infty)$ such that, for every $t \geq 0, S_{t}=\left(0, a^{*}(t)\right]$.

Proof of Lemma 4 We have to prove that, if $\mathcal{P}(t, y)=g(t, y)$ then $\mathcal{P}(t, x)=g(t, x)$ for all $x \leq y$. According to (2), we obtain that

$\mathbb{E}_{x}\left[e^{-r \tau}\left(\beta(t+\tau)-\gamma(t+\tau) V_{\tau}\right)\right] \leq \mathbb{E}_{y}\left[e^{-r \tau}\left(\beta(t+\tau)-\gamma(t+\tau) V_{\tau}\right)\right]+\mathbb{E}_{y-x}\left[e^{-r \tau} \gamma(t+\tau) V_{\tau}\right]$

The positive supermartingale $\left(e^{-r t} V_{t}\right)_{0 \leq t \leq \infty}$ admits zero as a last element and thus Optional Sampling Theorem yields $\mathbb{E}_{x}\left(e^{-r \tau} V_{\tau}\right) \leq x$ for every $\tau$ and every $x>0$. Using the fact that the positive function $\gamma$ is decreasing, we get

$$
\mathbb{E}_{y-x}\left[e^{-r \tau} \gamma(t+\tau) V_{\tau}\right] \leq \gamma(t)(y-x)
$$

Now, using (47) and taking the supremum over $\tau$ in (46) yields

$$
\mathcal{P}(t, x) \leq \mathcal{P}(t, y)+\gamma(t)(y-x)
$$

Since $y \in S_{t}$, we obtain $\mathcal{P}(t, y)=\beta(t)-\gamma(t) y$ and thus the inequality

$$
\mathcal{P}(t, x) \leq \beta(t)-\gamma(t) x .
$$

We then deduce that $x \in S_{t}$ since, by definition, $\mathcal{P}(t, x) \geq \beta(t)-\gamma(t) x$. Therefore, it follows that $S_{t}$ is an interval $\left(0, a^{*}(t)\right]$ where $a^{*}(t)$ is defined as $\sup \{x \in(0, \infty):(t, x) \in S\}$. Moreover, the optimal stopping time $\tau^{*}$ can be expressed as

$$
\tau^{*}=\inf \left\{s \geq 0: V_{s} \leq a^{*}(t+s)\right\} .
$$

We now show that the function $a^{*}$ is not constant.

Lemma 5 The optimal boundary function $a^{*}$ is not constant. 
Proof of Lemma 5 Because $\Phi(t)$ and $\Phi(t)$ are decreasing and tend to 0 when $t$ goes to infinity, we have $\beta(t) \leq 0$, for all $t \geq \bar{t}$, where $\bar{t}$ is implicitly defined by the equation

$$
C \frac{\bar{\Phi}(\bar{t})}{\bar{\Phi}(0)}+F \frac{\Phi(\bar{t})}{\bar{\Phi}(0)}-C \theta=0
$$

Thus, for all $t \geq \bar{t}, g(t, x)=\beta(t)-\gamma(t) x \leq 0$, for all $x>0$. Therefore, for all $t \geq \bar{t}$, $\mathcal{P}(t, x) \leq 0$ as the supremum of non positive real numbers. Now, for $s \geq 0$, we have

$$
\begin{aligned}
\mathcal{P}(t, x) & \geq e^{-r s} \beta(s+t)-\gamma(s+t) \mathbb{E}_{x}\left[e^{-r s} V_{s}\right] \\
& =e^{-r s} \beta(s+t)-\gamma(s+t) x e^{-\delta s} .
\end{aligned}
$$

Letting $s$ tend to infinity yields $\mathcal{P}(t, x) \geq 0$. Therefore, $\mathcal{P}(t, x)=0$ for all $t \geq \bar{t}$ which implies that $S_{t}=\emptyset$ for all $t \geq \bar{t}\left(\tau^{*}=\infty\right)$, or, equivalently, that $a^{*}(t)=0$ for all $t \geq \bar{t}$.

We now show that $S \neq \emptyset$, from which it will result that there exists $t \in[0, \bar{t})$ such that $a^{*}(t)>0$. This will end the proof of Lemma 5 . We proceed by way of contradiction. Assume $S=\emptyset$, then $\tau=\infty$ is optimal for problem (41) such that we have $\mathcal{P}(t, x)=0$ for all $t \geq 0$ and for all $x>0$. This implies in particular that, for all $x>0$,

$$
\mathcal{P}(0, x)=P(x)=0 \geq \beta(0)-\gamma(0) x=\int_{0}^{\infty} e^{-r s}\left(C \frac{\bar{\Phi}(s)}{\bar{\Phi}(0)}+F \frac{\Phi(s)}{\bar{\Phi}(0)}-\theta C\right) d s-x,
$$

where the right hand side of (50) is the payoff function of problem (42) according to the stopping strategy $\tau=0$. Now, it follows from assumption (13) that Equation (50) cannot be satisfied for all $x>0$. This yields a contradiction. Therefore, $S \neq \emptyset$ and $a^{*}(t)>0$ for some $t \in[0, \bar{t})$.

Lemma 6 The strategy $\tau_{a^{*}(.)}$ is not an equilibrium default policy.

Proof of Lemma 6 Let us assume that equity holders follow the strategy $\tau_{a^{*}(.)}$. Then, on the set $\left\{\tau_{a^{*}(.)}>t\right\}$ the equity value is

$$
\mathbb{E}\left[\int_{t}^{\tau_{a^{*}(.)}} e^{-r(s-t)}\left(\delta V_{s}-(1-\theta) C-f+d\left(s, \tau_{a^{*}(.)}\right)\right) d s \mid \mathcal{F}_{t}\right] .
$$

Now, from Lemma 5 , we know that $a^{*}(t)=0$ for all $t \geq \bar{t}$. We then deduce that $\tau_{a^{*}(t+.)}=\infty$ for all $t \geq \bar{t}$ and thus equity holders issue riskless debt yielding a value of newly issued debt equal to ${ }^{18}$

$$
f d s \int_{0}^{\infty}\left(\frac{c}{r}\left(1-e^{-r u}\right)+e^{-r u}\right)[-d \Phi(u)] .
$$

However, never going default for $t \geq \bar{t}$ is clearly sub-optimal since the associated equity value

$$
\mathbb{E}\left[\int_{0}^{\infty} e^{-r s}\left(\delta V_{s}-(1-\theta) C-f+f \int_{0}^{\infty}\left(\frac{c}{r}\left(1-e^{-r u}\right)+e^{-r u}\right)[-d \Phi(u)]\right) d s\right]
$$

\footnotetext{
${ }^{18}$ This is easily deduced from (9)
} 
would be negative for a current value of the firm's asset sufficiently small ${ }^{19}$. The strategy $\tau_{a^{*}(.)}$ does not satisfy Proposition 1. It is not an equilibrium default policy. This concludes the proof of Proposition 3.

\section{Appendix $\mathrm{C}$}

The following lemma completes the proof of Lemma 1

Lemma 7 The cash flow to equity $g_{B}(x)=\delta x-(1-\theta) C-f+d_{B}(x)$ is increasing over $(0, \infty)$.

To show that the function $x \longrightarrow d_{B}(x)$ is increasing over $(B, \infty)$ we prove that the value $b_{B}(x)$ of a bond with face value 1 , maturity $u$ and default time $\tau_{B}$ increases with the current value of the firm's asset $x$. When the default policy is a constant barrier strategy $\tau_{B}$, we have

$$
b_{B}(x)=\frac{c}{r}+\left(1-\frac{c}{r}\right) e^{-r u}\left(1-K_{B}(u, x)\right)+\left(\frac{1}{F}(1-\alpha) B-\frac{c}{r}\right) G_{B}(u, x)
$$

where

$$
K_{B}(u, x)=\int_{0}^{u} k_{B}(s, x) d s, G_{B}(u, x)=\int_{0}^{u} e^{-r s} k_{B}(s, x) d s
$$

and where $k_{B}(s, x)$ is the density function of the first passage time of the process $V_{s}$ to the barrier $B$. A standard computation shows that the density $k_{B}$ decreases with the current value of the firm's asset $x$. Then, the computation of the derivative of $b_{B}$ yields the relations

$$
\begin{aligned}
b_{B}^{\prime}(x) & =-\left(1-\frac{c}{r}\right) e^{-r u} \frac{\partial K_{B}}{\partial x}(u, x)-\left(\frac{c}{r}-\frac{1}{F}(1-\alpha) B\right) \frac{\partial G_{B}}{\partial x}(u, x) \\
& \geq\left(1-\frac{c}{r}\right)\left(-e^{-r u} \frac{\partial K_{B}}{\partial x}(u, x)+\frac{\partial G_{B}}{\partial x}(u, x)\right) \\
& \geq 0,
\end{aligned}
$$

where Equation (52) comes from the inequality $B \leq \frac{F}{1-\alpha}$ and the last inequality from the relation $^{20} 1<\frac{c}{r}$ together with the fact that

$$
\begin{aligned}
& -e^{-r u} \frac{\partial K_{B}}{\partial x}(u, x)+\frac{\partial G_{B}}{\partial x}(u, x) \\
= & -e^{-r u} \int_{0}^{u} \frac{\partial k_{B}}{\partial x}(s, x) d s+\int_{0}^{u} e^{-r s} \frac{\partial k_{B}}{\partial x}(s, x) d s<0
\end{aligned}
$$

\section{Proof of Lemma 2.}

We use the relation

$$
E_{B}(x)=\mathbb{E}_{x}\left[\int_{0}^{\tau_{B}} e^{-r s} h\left(s, V_{s}\right) d s\right]=v_{B}(x)-D_{B}(x)
$$

\footnotetext{
${ }^{19}$ An easy computation shows that the inequality $\int_{0}^{\infty} e^{-r u}[-d \Phi(u)]>1-r \bar{\Phi}(0)$ and the condition $F>\frac{\theta C}{r}$ imply that $-(1-\theta) C-f+f \int_{0}^{\infty}\left(\frac{c}{r}\left(1-e^{-r u}\right)+e^{-r u}\right)[-d \Phi(u)]<0$.

${ }^{20}$ Following the literature, we consider that the total debt is issued at par. At issuance its value $F$ is lower than a risk-less bond with infinite maturity and coupon payment $C$. This yields the inequality $F<\frac{C}{r}$ or equivalently $1<\frac{c}{r}$.
} 
where the expressions for $v_{B}(x)$ and $D_{B}(x)$ are deduced from Equations (14) and (11) when the default policy is a constant barrier strategy $\tau_{B}$. We have

$$
v_{B}(x)=x+\frac{\theta C}{r}\left(1-\int_{0}^{\infty} e^{-r s} k_{B}(s, x) d s\right)-\alpha B \int_{0}^{\infty} e^{-r s} k_{B}(s, x) d s
$$

and

$$
\begin{aligned}
D_{B}(x)= & \int_{0}^{\infty} f \Phi(s)\left(\frac{c}{r}+\left(1-\frac{c}{r}\right) e^{-r s}\right) d s-\int_{0}^{\infty} f \Phi(s)\left(1-\frac{c}{r}\right) e^{-r s} K_{B}(s, x) d s \\
& +\int_{0}^{\infty} f \Phi(s)\left(\frac{1}{F}(1-\alpha) B-\frac{c}{r}\right) G_{B}(s, x) d s
\end{aligned}
$$

where functions $k_{B}, K_{B}$ and $G_{B}$ were introduced in Lemma 7 . The expressions for $K(s, x)$ and for $G(s, x)$ are standard and can be found for instance in Leland and Toft [22] page 990. We have

$$
\begin{aligned}
K_{B}(s, x) & =N\left(h_{1}(s, x ; B)\right)+\left(\frac{x}{B}\right)^{-2 a} N\left(h_{2}(s, x ; B)\right) \\
G_{B}(s, x) & =\left(\frac{x}{B}\right)^{-a+z} N\left(q_{1}(s, x ; B)\right)+\left(\frac{x}{B}\right)^{-a-z} N\left(q_{2}(s, x ; B)\right)
\end{aligned}
$$

where

$$
\begin{aligned}
& q_{1}(s, x ; B)=-\frac{1}{\sigma \sqrt{s}} \ln \left(\frac{x}{B}\right)-z \sigma \sqrt{s}, q_{2}(s, x ; B)=q_{1}(s, x ; B)+2 z \sigma \sqrt{s}, \\
& h_{1}(s, x ; B)=-\frac{1}{\sigma \sqrt{s}} \ln \left(\frac{x}{B}\right)-a \sigma \sqrt{s}, h_{2}(s, x ; B)=h_{1}(s, x ; B)+2 a \sigma \sqrt{s}, \\
& a=\frac{r-\delta-\frac{\sigma^{2}}{2}}{\sigma^{2}}<z=\frac{\left(a^{2} \sigma^{4}+2 r \sigma^{2}\right)^{\frac{1}{2}}}{\sigma^{2}}
\end{aligned}
$$

and where $N$ is the cumulative distribution function of a standard normal law. Then, taking the derivative of expression $E_{B}(x)=v_{B}(x)-D_{B}(x)$ one gets, $B E^{\prime}\left(B^{+}\right)=k_{1}+k_{2} B$ where

$$
k_{2}=1+\alpha(a+z)-(1-\alpha) \int_{0}^{\infty} \frac{f}{F} \Phi(s)\left((-a+z)-\Gamma_{z}(s)\right) d s
$$

where $\Gamma_{z}(s)=2 z N(z \sigma \sqrt{s})+\frac{2}{\sigma \sqrt{s}} n(z \sigma \sqrt{s})$ and $n(x)=\frac{1}{\sqrt{2 \pi}} e^{-\frac{1}{2} x^{2}}$, and

$$
k_{1}=\frac{\theta C}{r}(a+z)+\int_{0}^{\infty} f \Phi(s) \frac{c}{r}\left(-a+z-\Gamma_{z}(s)\right) d s+\int_{0}^{\infty} f \Phi(s)\left(\frac{c}{r}-1\right) e^{-r s} \Gamma_{a}(s) d s .
$$

Because $\Gamma_{z}(s) \geq 2 z$, the real number $k_{2}$ is clearly positive and more precisely, $k_{2} \geq 1+(a+$ $z) \geq 0$.

It remains to check that $k_{1}<0$. Using equalities $e^{-r s} n(a \sigma \sqrt{s})=n(z \sigma \sqrt{s})$ and $C=$ $\int_{0}^{\infty} f c \Phi(s) d s=c F$ we obtain that

$$
\begin{aligned}
k_{1}= & \frac{C}{r}(\theta(a+z)+z-a)+\left(\frac{c}{r}-1\right) \int_{0}^{\infty} f \Phi(s)\left(2 a e^{-r s} N(a \sigma \sqrt{s})-2 z N(z \sigma \sqrt{s})\right) d s \\
& -\int_{0}^{\infty} f \Phi(s) \Gamma_{z}(s) d s .
\end{aligned}
$$


The function $s \rightarrow 2 a e^{-r s} N(a \sigma \sqrt{s})-2 z N(z \sigma \sqrt{s})$ is decreasing and thus bounded by $a-z$. Therefore, using $\Gamma_{z}(s) \geq 2 z$ for all $s \geq 0$ together with the condition $F>\frac{\theta C}{r}$, we deduce from (54) that $k_{1}<0$.

Finally, we show that the inequality $B_{\infty}<\frac{F}{1-\alpha}$ implies $B^{*} \equiv \frac{-k_{1}}{k_{2}}<\frac{F}{1-\alpha}$. Note that the threshold $B_{\infty}$ is solution to Equation (7) and satisfies $B_{\infty}=(1-\theta) \frac{C}{r} \frac{a+z}{1+a+z}$. Using (53) and (54) one obtains

$$
\begin{aligned}
k_{2} F+(1-\alpha) k_{1}= & F(1+\alpha(a+z))+(1-\alpha) \frac{\theta C}{r}(a+z)+(1-\alpha)\left(\frac{c}{r}-1\right)(-a+z) F \\
& -(1-\alpha)\left(\frac{c}{r}-1\right) \int_{0}^{\infty} f \Phi(s)\left(2 z N(z \sigma \sqrt{s})-2 a e^{-r s} N(a \sigma \sqrt{s})\right) d s \\
> & F(1+\alpha(a+z))+(1-\alpha) \frac{\theta C}{r}(a+z)+(1-\alpha)\left(\frac{c}{r}-1\right)(-a+z) F \\
& -2 z(1-\alpha)\left(\frac{c}{r}-1\right) F \\
= & (1+a+z) F-(1-\alpha)(a+z)(1-\theta) \frac{C}{r} .
\end{aligned}
$$

The result follows.

\section{References}

[1] Bielecki, T.R., and Rutkowski, M.: Credit Risk Modeling, Valuation and Hedging. Springer-Verlag Berlin Heidelberg, (2004)

[2] Chen, N., and Kou, S.: Credit spreads, optimal capital structure, and implied volatility with endogenous default and jump risk. Mathematical Finance, 19, 3, 343-378, (2009)

[3] Dayanik, S., and Karatzas, I.: On the optimal stopping problem for one-dimensional diffusions. Stoch. Process. Appl, 107, 173-212, (2003)

[4] Duffie, D., Dynamic Asset Pricing Theory. Princeton University Press, Third Edition, (2001)

[5] Duffie, D., and Lando, D.: Term structures of credit spreads with incomplete accounting information. Econometrica, 69, 633-664, (2001)

[6] El Karoui, N.: Les aspects probabilistes du contrôle stochastique. Lecture Notes in Mathematics 876,74-239. Springer, Berlin, (1981)

[7] Eom, Y.H., J. Helwege, and J.Z. Huang.: Structural models of corporate bond pricing: An empirical analysis. Rev. Financ. Stud. 17, 499-544, (2004)

[8] Ericsson, J. and Renault, O.: Liquidity and credit risk. J. Finance, 61, 2219-2250, (2006)

[9] Fan, H. and Sundaresan, S.: Debt valuation, renegotiation, and optimal dividend policy. Rev. Financ. Stud. 13, 1057-1099, (2000) 
[10] Friedmann, A. Stochastic Differential Equations and Applications, Vol 2. Academic Press, (1975)

[11] Goldstein, R., N. Ju, and H. Leland.: An EBIT-based model of dynamic capital structure. J. Business 74(4), 483-512, (2001)

[12] Hackbarth, D., J. Miao, and E. Morellec.: Capital strucutre, credit risk, and macroeconomic conditions. J. Fin. Economics, 82, 519-550, (2006)

[13] Harsanyi, J. and Selten, R.: A general Theory of Equilibrium Selection in Games, MIT Press (1988)

[14] Hilberink, B., Rogers, L.C.G.: Optimal capital structure and endogenous default. Finance Stochast. 6, 237-263, (2002)

[15] Karatzas, I. and Shreve, S. Methods of mathematical finance. Springer, Berlin, (1998)

[16] Kyprianou, A.E., Surya, B.A.: Principles of smooth and continuous fit in the determination of endogenous bankruptcy levels. Finance Stochast. 11, 131-152, (2007)

[17] Lando, D.: Credit Risk Modeling Theory and Applications. Princeton University Press. (2004)

[18] Leland, H.E.: Corporate debt value, bond covenants, and optimal capital structure. J. Finance 49, 1213-1252, (1994)

[19] Leland, H.E.: Bond prices, yield spreads, and optimal capital structure with default risk, Working paper 240, IBER, University of California, Berkeley, (1994)

[20] Leland, H.E.: Agency costs, risk management, and capital structure. J. Finance 53, 4, 1213-1243, (1998)

[21] Leland, H.E.: Predictions of Default Probabilities in Structural Models of Debt. J. Invest. Manag. 2, 2, 5-20, (2004).

[22] Leland, H.E., Toft, K.B.: Optimal capital structure, endogeneous bankruptcy, and the term structure of credit spreads. J. Finance 51, 987-1019 (1996)

[23] Mauer, D. and Ott, S.: Agency costs, underinvestment, and optimal capital structure. In M. Brennan, L. Trigeorgis, eds., Project flexibility, agency, and competition: New developments in the theory and application of real options. Oxford, 151-179, (2000)

[24] Mella-Barral, P. and Perraudin,W.: Strategic debt service. J. Finance 52, 531-556, (1997)

[25] Øksendal, B. Stochastic Differential Equations: An Introduction with Applications, 5th ed. Springer, Berlin, (1998)

[26] Revuz, D. and Yor, M. Continuous Martingales and Brownian Motion, third ed. Springer, Berlin, (1998) 
[27] Villeneuve, S.: On the threshold strategies and smooth-fit principal for optimal stopping problems. J. Appl. Prob 44, 181-198 (2007) 